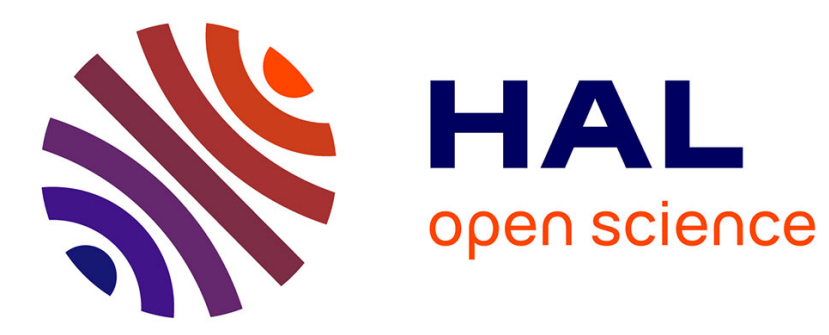

\title{
Plasmonic topological metasurface by encircling an exceptional point
}

\author{
Qinghua Song, Mutasem Odeh, Jesús Zúñiga-Pérez, Boubacar Kanté, Patrice
} Genevet

\section{- To cite this version:}

Qinghua Song, Mutasem Odeh, Jesús Zúñiga-Pérez, Boubacar Kanté, Patrice Genevet. Plasmonic topological metasurface by encircling an exceptional point. Science, 2021, 373 (6559), pp.1133-1137. 10.1126/science.abj3179 . hal-03357725

\section{HAL Id: hal-03357725 \\ https://hal.science/hal-03357725}

Submitted on 30 Sep 2021

HAL is a multi-disciplinary open access archive for the deposit and dissemination of scientific research documents, whether they are published or not. The documents may come from teaching and research institutions in France or abroad, or from public or private research centers.
L'archive ouverte pluridisciplinaire HAL, est destinée au dépôt et à la diffusion de documents scientifiques de niveau recherche, publiés ou non, émanant des établissements d'enseignement et de recherche français ou étrangers, des laboratoires publics ou privés. 


\title{
Title: Plasmonic Topological Metasurface by Encircling an Exceptional Point Short title: Exceptional Topological Metasurface
}

Authors: Qinghua Song ${ }^{1}$, Mutasem Odeh² ${ }^{2}$ Jesús Zúñiga-Pérez ${ }^{1}$, Boubacar Kanté2 ${ }^{2}$ and Patrice Genevet $^{1 *}$

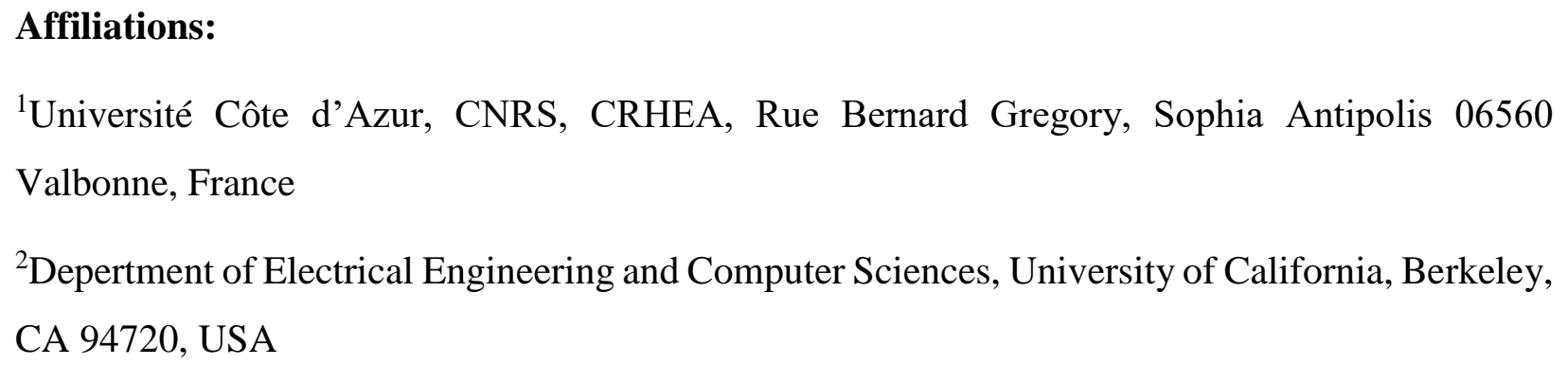

${ }^{1}$ Université Côte d'Azur, CNRS, CRHEA, Rue Bernard Gregory, Sophia Antipolis 06560 Valbonne, France

${ }^{2}$ Depertment of Electrical Engineering and Computer Sciences, University of California, Berkeley, CA 94720, USA

*Correspondence to: Patrice.Genevet@ crhea.cnrs.fr.

\begin{abstract}
Resonant scattering, guided mode propagation phase and/or orientation-dependent phase retardations are the three main mechanisms used until now to conceive optical metasurfaces.

15 Here we introduce an additional degree of freedom to address optical phase engineering by exploiting the topological features of non-Hermitian matrices operating near their singular points. Choosing metasurface building blocks to encircle a singularity following an arbitrarily closed trajectory in parameter space, we engineer topologically-protected full $2 \pi-$ phase on a specific reflected polarization channel. The ease of implementation together with its compatibility with other phase-addressing mechanisms bring topological properties into the realm of industrial applications at optical frequencies, and prove that metasurface technology represents a convenient test bench to study and validate topological photonic concepts.
\end{abstract}

One Sentence Summary: Topology-protected $2 \pi$ phase by encircling exceptional point in arbitrary closed path for unique metasurface wavefront shaping. 
Metasurfaces (1-6) are artificial materials constructed with subwavelength arrays of spatiallydistributed nanostructures that enable versatile wave front engineering. From a fundamental perspective, a metasurface is an open system, continuously exchanging and/or absorbing energy between the impinging field and the resonant nanostructures. Unlike closed systems, open systems experience a phase transition at the exceptional point (EP) leading to complex scattering phenomena related to optical singularities (7). The latter can be equivalently described in an optical system using the scattering matrix and Hamiltonian representations (8-10). Singularities also manifest in the reflection-zero regime, perfect absorption and bound state in the continuum, giving rise to unidirectional transmission or reflection $(11,12)$, single mode laser operation (13), extreme sensitiveness to perturbation $(14,15)$, etc. Tailoring light reflection, or transmission, with metasurfaces consists in addressing the values of complex coefficients by varying the nanostructure geometries. Choosing any arbitrary loop in parameter space to achieve phase distribution does not necessarily lead to full $2 \pi$ phase accumulation. Here we show how to leverage on the $2 \pi$-phase excursion occurring on the coefficient value by encircling zeros/singularities in the complex plane. Encircling singular point to accumulate an optical phase difference is of crucial interest for wavefront engineering, enrichening the metasurface design toolbox with an additional phase addressing mechanism.

Previous works on metasurfaces have shown that non-Hermitian metasurfaces can operate close to an EP, and have approached the singularity thanks to a series of individually-distinct metasurfaces for which a given parameter is changed (16-19). The exploitation of the robust topological phase around the EP, referred to as exceptional topological (ET) phase, to realize functional devices has remained elusive. The proposed ET-phase is fundamentally different from conventional Pancharatnam-Berry (PB) phase (20-23) and, as a consequence, ET can be combined with other phase addressing mechanisms, including PB, to achieve full and independent control of the polarization channels. We demonstrate a meta-hologram using the ET phase encircling the EP to project an image of "C" for left circular polarized (LCP) light, leaving the cross right circular polarized (RCP) channel unaffected. By combining the ET and PB phases, we decouple the two circular polarizations $(\mathrm{CP})$ and achieve two different holographic projections with an image of "A" for LCP light and "B" for RCP light.

30 The plasmonic topological metasurface consists of three layers, where a dielectric layer is sandwiched between a metal ground and a metallic structured layer, as shown in Fig. 1A. The 
metal ground blocks all the transmission so that the metasurface works in the reflective regime. The structured layer is formed by a two-dimensional (2D) planar chiral structure array, which is composed of an "L" shaped nanostructure, near-field coupled to a straight nano rod, representing a Hangul syllable of "느". To observe the EP complex-square-root topology of the eigenvalues surface, two parameters need to be varied, defining thereby the parameter space $\boldsymbol{R}=\left(L_{1}, L_{3}\right)$, with $L_{1}$ and $L_{3}$ being the geometrical parameters shown in Fig. 1A. $L_{1}$ and $L_{3}$ are chosen as they play key roles in the $y$ - and $x$-polarized resonances, as shown in Supplementary fig. S9 and Note 4, giving access to a full $2 \pi$ reflection phase for $r_{+-}$(where the subscript +- represent reflected CP conversion from RCP $(|-\rangle)$ to LCP $(|+\rangle))$. The EP is obtained by adjusting the nanostructure structural properties, so that both eigenstates degenerate as CP beams. If the degenerate eigenstate is $\mathrm{RCP}$, i.e., $\hat{r}|-\rangle=\lambda_{1,2}|-\rangle$, where $\hat{r}$ is the reflection matrix and $\lambda_{1}=\lambda_{2}$ are two degenerate eigenvalues, then RCP input light is preserved, and the CP conversion channel from RCP to LCP vanishes, i.e., $r_{+-}=0$. A zero point or singularity point, equivalent to perfect absorption cases (24-26), is obtained for $r_{+-}$as shown in Fig. 1B. The chiral response of the surface current distribution at the EP, shown in the Supplementary fig. S7, is a key ingredient to achieve circular polarization dependent reflectivity. The induced chirality is an inherent property of the EP from a symmetric non-Hermitian Hamiltonian (27) and leads to an asymmetric conversion from one circular polarization to the other and vice versa (i.e., $r_{+-} \neq r_{-+}$). Interestingly, the spectral response of only one of the two CP conversion undergoes a $2 \pi$ phase change around the EP 20 wavelength. Due to the topological protection provided by encircling of the EP, as discussed in Supplementary Note 1 and 2 , the $2 \pi$ phase accumulation is maintained for the reflected phase map irrespective of the closed path, as long as it encircles one EP in parameter space. Given the metasurface versatility, we realize on a single component an assembly of antennas with different parameters chosen so as to move around the EP in the parameter space, thus realizing simultaneously a set of optical responses (see Fig. 1G and 1J). Amplitude variation with singular value is also observed. The other reflective CP conversion coefficient $\left(r_{-+}\right)$does not present a singularity in this parameter space, resulting in an almost constant phase and amplitude profiles as shown in Fig. 1C.

The conventional PB phase works oppositely for the two reflective CP conversion beams, as reported in Fig. 1D-1F. Being fundamentally different, ET phase is imposed on only one CP 
conversion and thus can be combined with the standard PB phase (see more details in Supplementary Note 5). As a result, the combination of ET and PB phases can be used to decouple the phase of the two CP conversion beams, leading to independent control of each polarization converted wavefront, as shown in Fig. 1G-1L.

A spectral singularity point of $r_{+-}$, together with its degeneracy of the eigenvalues and eigenstates at $\lambda=600 \mathrm{~nm}$ proof the existence of an EP as shown by the red star in Fig. 2. This behavior is further confirmed by the intersecting double Riemann surface corresponding to the reflection matrix eigenvalues as shown in Fig. 3A and 3B. Various phase and amplitude-conversion distribution choices are possible for the deflecting unit-cell. Here we chose a simple unit-cell phase coverage, i.e., considering $90^{\circ}$ phase steps, so as to keep the distance between neighboring antennas constant, which slightly compromises the amplitude of the third structure as shown in Fig. 3E. Seven designs of PB phase interval of $60^{\circ}$ are also presented in Fig. 3C-3H for the combination with ET phase.

We experimentally demonstrate an ET-phase gradient metasurface, behaving as a beam deflector and a meta-hologram. The scanning electron micrograph (SEM) of the fabricated beam deflector is shown in Fig. 4A. An asymmetric reflection of the two CP conversion beams is measured in Fig. 4B, with a maximum efficiency of $12 \%$ and power ratio around 10:1 as quantified in Fig. 4C. The spectral position of the singular design (EP antenna) evolves as a function of the antenna structural parameters, as shown in Fig. S13, indicating that small nanofabrication errors can 20 slightly shift the position of the EP and modify the expected deflection efficiencies and power ratio. To show the versatility of this wavefront phase encoding technique we realize a metahologram as fabricated in Fig. 4D. As expected, a holographic image of " $\mathrm{C}$ " is displayed for $r_{+-}$ as shown in Fig. 4E, but no image appears for $r_{-+}$as shown in Fig. 4F.

The wealth of applications of the ET phase expands by combining it with other phase-control mechanisms. Here, ET is combined with the widespread PB phase by simply rotating the metasurface elements, as shown in Fig. 4G-4L, to decouple the deflection angles of the two CP beams in Fig. 4H and 4I. Similarly, ET+PB phase encoding is applied to project two different CP holographic images as shown in Fig. 4K and 4L.

30 Concluding remarks. We have demonstrated a planar chiral plasmonic metasurface that exhibits $2 \pi$ topological phase accumulation in reflection regime by choosing nanoantenna designs 
distributed along any arbitrarily closed parameter loop encircling the EP. We have shown $2 \pi$-phase accumulation is imposed on only one of the CP conversion beams defined by the chirality of the encircled EP. Exploiting a linear combination of ET and PB phases we decoupled LCP and RCP channels. Addressing phase retardation by encircling singularities of the S-matrix open vast research opportunities for unusual polarization, linear and nonlinear topological electromagnetic field control at optical frequencies.

\section{References and Notes:}

1. N. Yu, et al., Science. 334, 333-337 (2011).

2. P. Genevet, et al., Nat. Nanotechn. 10, 804-809 (2015).

3. A. Alù, N. Engheta, Nat. Photonics 2, 307-310 (2008).

4. Q. Song, et al., Nat Commun. 12, 3631 (2021).

5. M. V. Gorkunov, A. A. Antonov, Y. S. Kivshar, Phys. Rev. Lett. 125, 093903 (2020),

6. D. B. Haim, L. Michaeli, O. Avayu, T. Ellenbogen, Opt. Express. 28, 17923 (2020).

15 7. W. D. Heiss, J. Phys. A: Math. Theor. 45, 444016 (2012).

8. W. R. Sweeney, C. W. Hsu, S. Rotter, A. D. Stone, Phys. Rev. Lett. 122, 093901 (2019).

9. $\quad$ Ş. K. Özdemir, S. Rotter, F. Nori, L. Yang, Nat. Mater. 18, 783-798 (2019).

10. M.-A. Miri, A. Alù, Science 363, 6422, (2019).

11. A. Regensburger, et al., Nature 488, 167-171 (2012).

12. Z. Lin, et al., Phys. Rev. Lett. 106, 213901 (2011).

13. L. Feng, Z. J. Wong, R.-M. Ma, Y. Wang, X. Zhang, Science 346, 972-975 (2014).

14. J.-H. Park, et al., Nat. Phys. 16, 462-468 (2020).

15. M. C. Rechtsman, Nature. 548, 161-162 (2017).

16. H. Alaeian, J. A. Dionne, Phys. Rev. A. 89, 033829 (2014).

25 17. X. Wang, X. Fang, D. Mao, Y. Jing, Y. Li, Phys. Rev. Lett. 123, 214302 (2019).

18. M. Lawrence, et al., Phys. Rev. Lett. 113, 093901 (2014).

19. S. H. Park, et al., Nanophotonics 9, 1031-1039 (2020).

20. D. Lin, P. Fan, E. Hasman, M. Brongersma, Science 345, 298-302 (2014). 
21. G. Zheng, et al., Nat. Nanotechn. 10, 308-312 (2015).

22. Q. Song, et al., Nat. Commun. 11, 2651 (2020).

23. Q. Song, et al., Sci. Adv. 7, eabe1112 (2021).

24. E. Plum, N. I. Zheludev, Appl. Phys. Lett. 106, 221901 (2015).

25. X. Yang, M. Li, Y. Hou, J. Du, F. Gao, Opt. Express 27, 6801 (2019).

26. A. Krasnok, et al., Adv. Opt. Photon. 11, 892-951 (2019).

27. W. D. Heiss, H. L. Harney, Eur. Phys. J. D. 17, 149-151 (2001).

28. M. Mcintyre, G. Cairns, Geom Dedicata. 46, 149-159 (1993).

29. W. Luo, S. Xiao, Q. He, S. Sun, L. Zhou, Adv. Opt. Mater. 3, 1102-1108 (2015).

10

\section{Acknowledgements}

Funding: P.G. acknowledges funding from the European Union's Horizon 2020 research and innovation programme (Grant agreement no. 639109). B.K. acknowledges funding from Office of Naval Research Young Investigator Award (no. N00014-17-1-2671), and the Laboratory Directed Research and Development Program of Lawrence Berkeley National Laboratory under US Department of Energy (no. DE-AC02-05CH11231). Author contributions: Q.S., J.Z.P. and P.G. conceived the initial ideas and carried out the experiment. Q.S. designed the structure and performed the numerical calculations. M.O. and B.K. fabricated the samples. P.G. supervised the research. All the authors wrote the manuscript. Competing interests: The authors declare no competing financial interest. Data and materials availability: All data needed to evaluate the conclusions in the paper are available in the manuscript or the supplementary materials.

\section{Supplementary Materials:}

Materials and Methods

Notes 1-5 

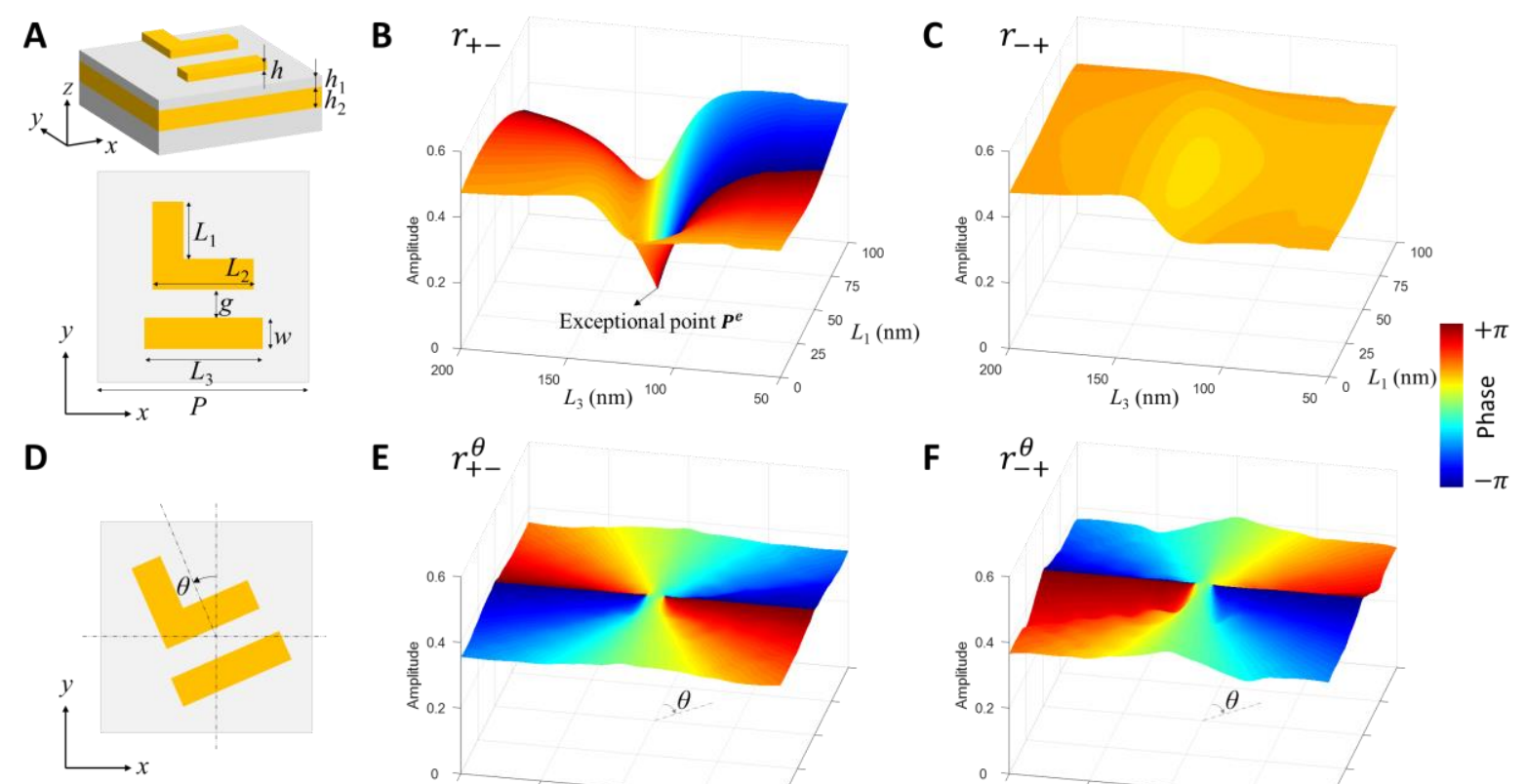

E $\quad r_{+-}^{\theta}$
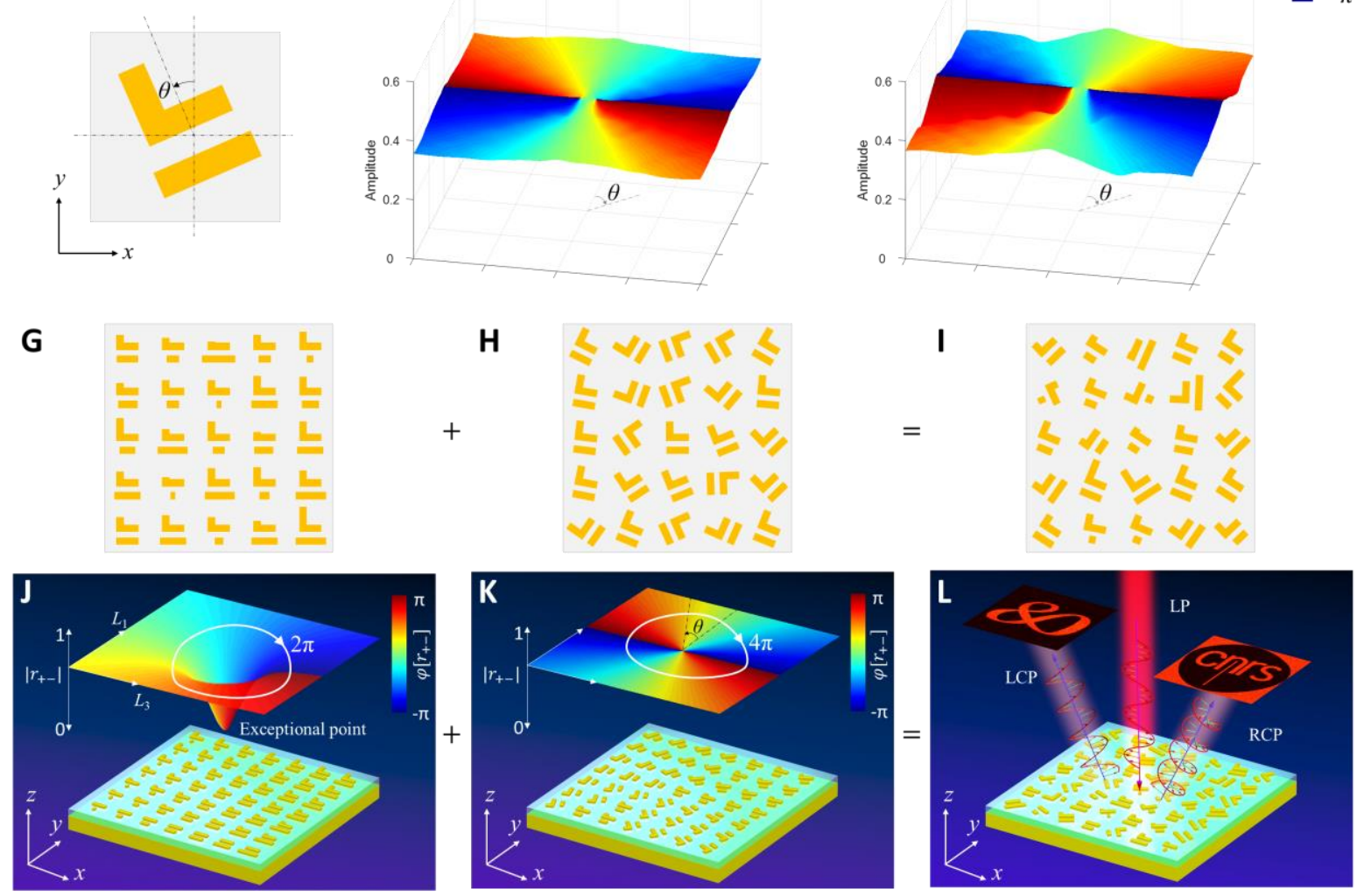

Fig. 1. Design principle of the topological metasurface. (A) Perspective (top panel) and top (bottom panel) view of $2 \mathrm{D}$ chiral meta-atom design in reflection. The dimension is $L_{2}=140 \mathrm{~nm}$, $P=300 \mathrm{~nm}, w=50 \mathrm{~nm}, g=70 \mathrm{~nm}, h=30 \mathrm{~nm}, h_{1}=40 \mathrm{~nm}, h_{2}=150 \mathrm{~nm}$. (B) Simulated CP conversion coefficients $r_{+-}$and (C) $r_{-+}$in the parameter space covered by $L_{1} \in[0,100 \mathrm{~nm}]$ and $L_{3} \in[50 \mathrm{~nm}, 200 \mathrm{~nm}]$. An EP is obtained at $\left(L_{1}, L_{3}\right)=(52 \mathrm{~nm}, 119 \mathrm{~nm})$. A vortex phase profile for $r_{+-}$and a topological protected $2 \pi$ phase accumulation is obtained by encircling the EP. (D) Top view of meta-atom with rotation angle of $\theta$. (E) Simulated CP conversion coefficients $r_{+-}^{\theta}$ and $(\mathrm{F}) r_{-+}^{\theta}$ with $\theta \in[0,2 \pi]$. In $\mathrm{E}$ and $\mathrm{F}$, the original ET phase without rotation $\left(r_{ \pm \mp}^{0}\right)$ has been subtracted to display PB phase only. The $z$-axis in B, C, E and F represents the amplitude and the color scale represents the phase. The combination of $(\mathrm{G})$ ET phase and $(\mathrm{H}) \mathrm{PB}$ phase is designed to realize an $\mathrm{ET}+\mathrm{PB}$ phase metasurface in (I). Schematics of the design implemented for combining the (J) ET and (K) PB phase control in (L) a single ET+PB phase metasurface, able to independently control the two CP beams. 

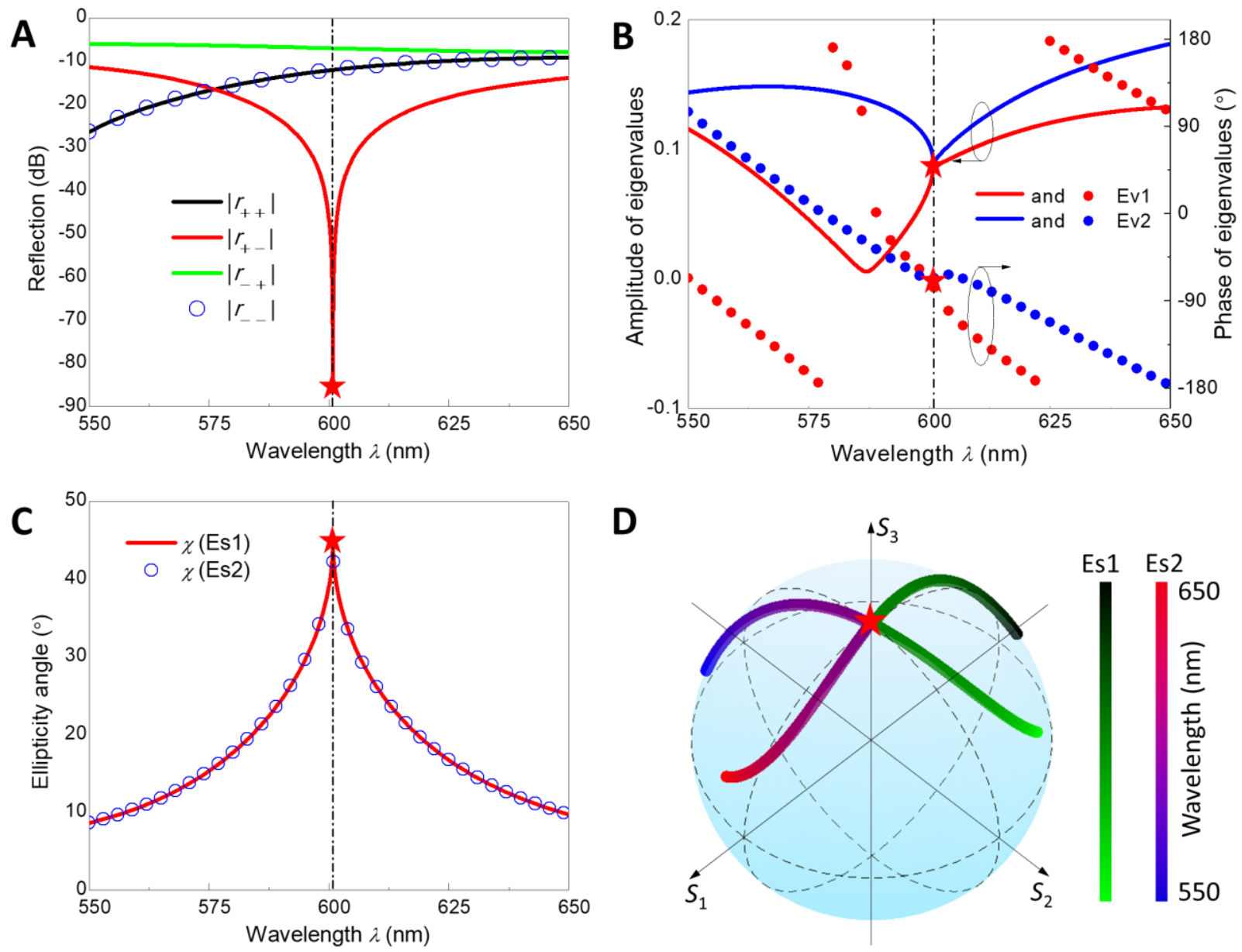

Fig. 2. Simulated reflection spectra and the corresponding eigenvalues and eigenstates. (A) Spectral dependence of the reflection matrix coefficients (in the circular basis). A singularity point, where $r_{+-}=0$, is observed at $\lambda=600 \mathrm{~nm}$. (B) Amplitude and phase of the reflection matrix eigenvalues. The two eigenvalues degenerate at $\lambda=600 \mathrm{~nm}$. (C) Simulated ellipticity angle of the two eigenstates, which overlap and degenerate as RCP at $\lambda=600 \mathrm{~nm}$. (D) Position of the eigenstates on the Poincaré sphere as a function of wavelength, which degenerate at $\lambda=600 \mathrm{~nm}$. All the evidences prove that an EP is obtained at $\lambda=600 \mathrm{~nm}$. 
A
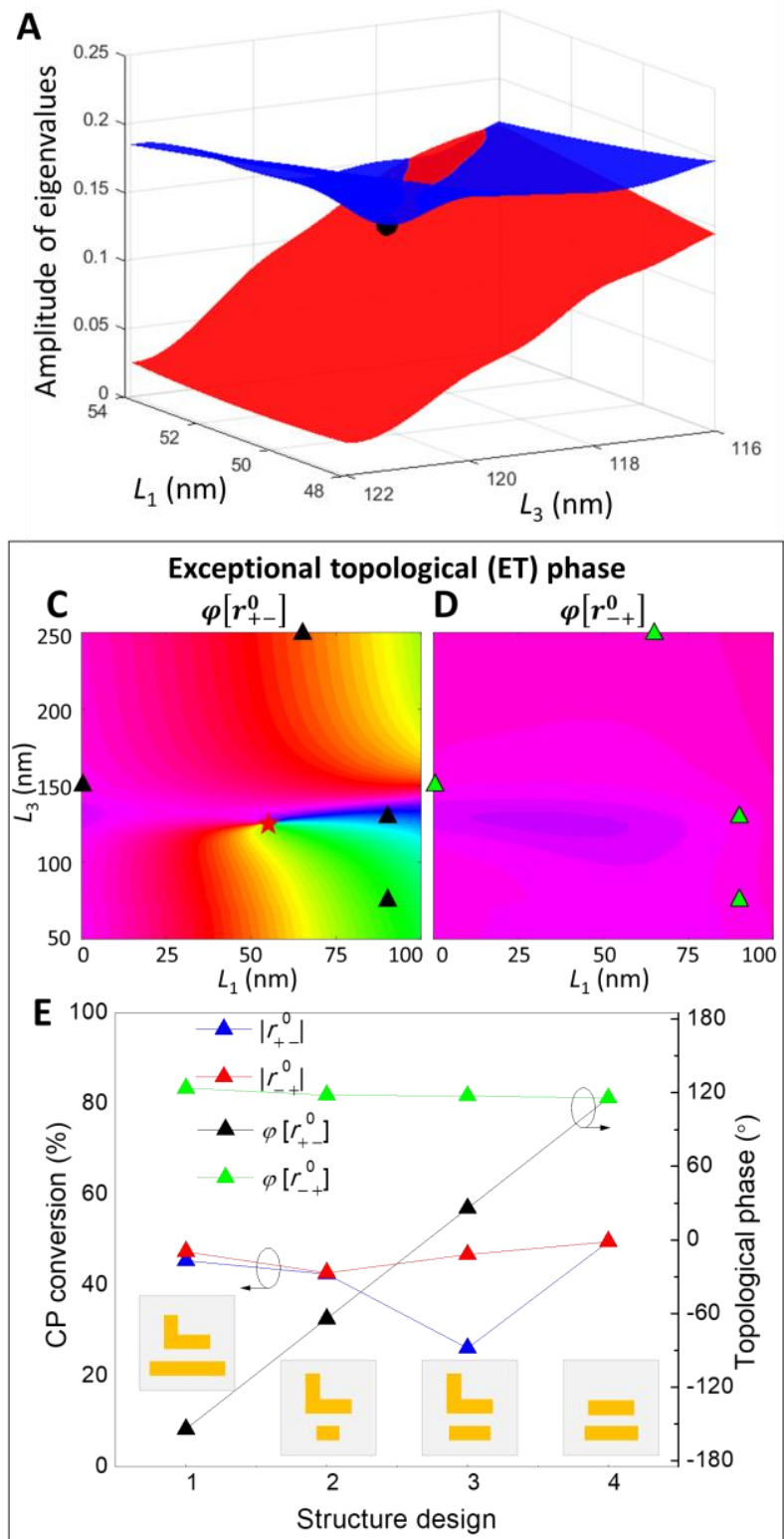

B

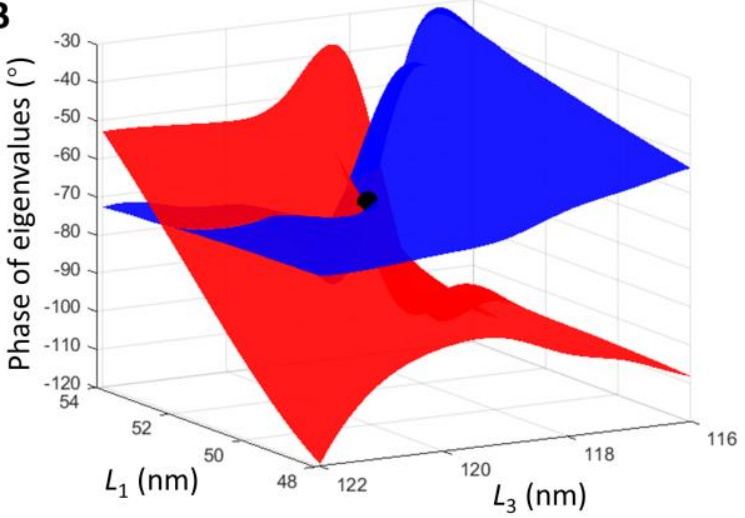

Pancharatnam-Berry (PB) phase
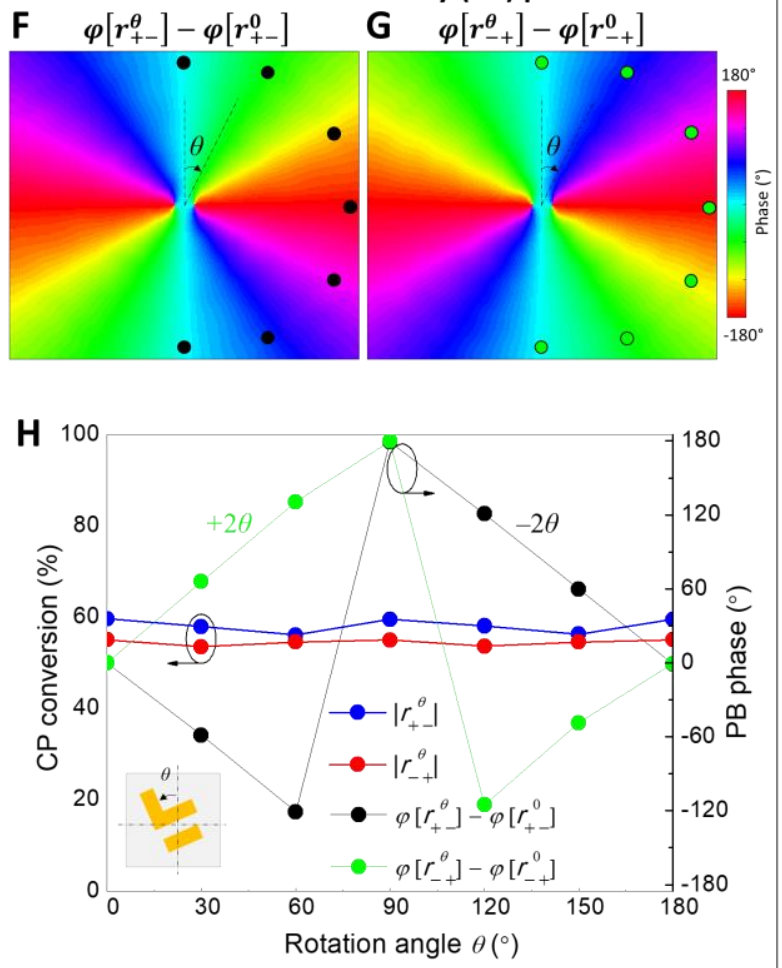

Fig. 3. Simulation results of the metasurface in the parameter space $R=\left(L_{1}, L_{3}\right)$. (A) Simulated amplitude and (B) phase of two eigenvalues at $\lambda=600 \mathrm{~nm}$. A self-intersecting Riemann surface profile is observed. The EP is highlighted as black dot, where two eigenvalues degenerate at $\left(L_{1}, L_{3}\right)=(52 \mathrm{~nm}, 119 \mathrm{~nm})$. (C) Simulated ET phase of $r_{+-}^{0}$ and (D) nearly constant phase of $r_{-+}^{0}$. (E) Four meta-atom designs are selected such that the ET phase interval is $90^{\circ}$, highlighted as triangles in $\mathrm{C}$ and $\mathrm{D}$. The inset figures are four corresponding structure designs. (F) Simulated PB phase of $r_{+-}^{\theta}$ and $(\mathrm{G}) r_{-+}^{\theta}$, where the presented phase has been subtracted by the original phase of $r_{ \pm \mp}^{0}$ without rotation to display PB phase only. $(\mathrm{H})$ Seven rotated structures are presented with rotation angle from $0^{\circ}$ to $180^{\circ}$ stepped by $30^{\circ}$, highlighted as dots in $\mathrm{F}$ and $\mathrm{G}$. (See more details in Supplementary fig. S11 and S14). 

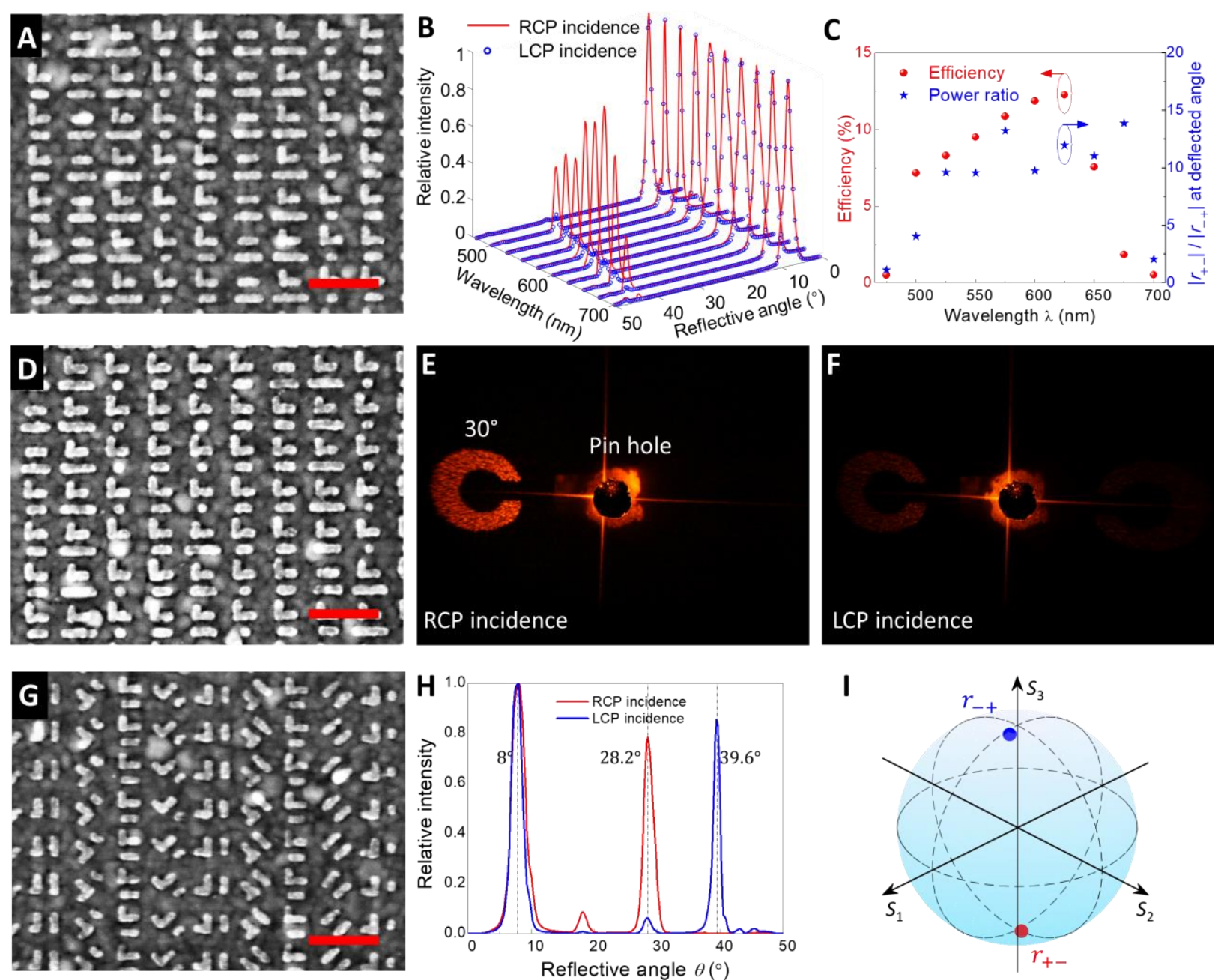

I
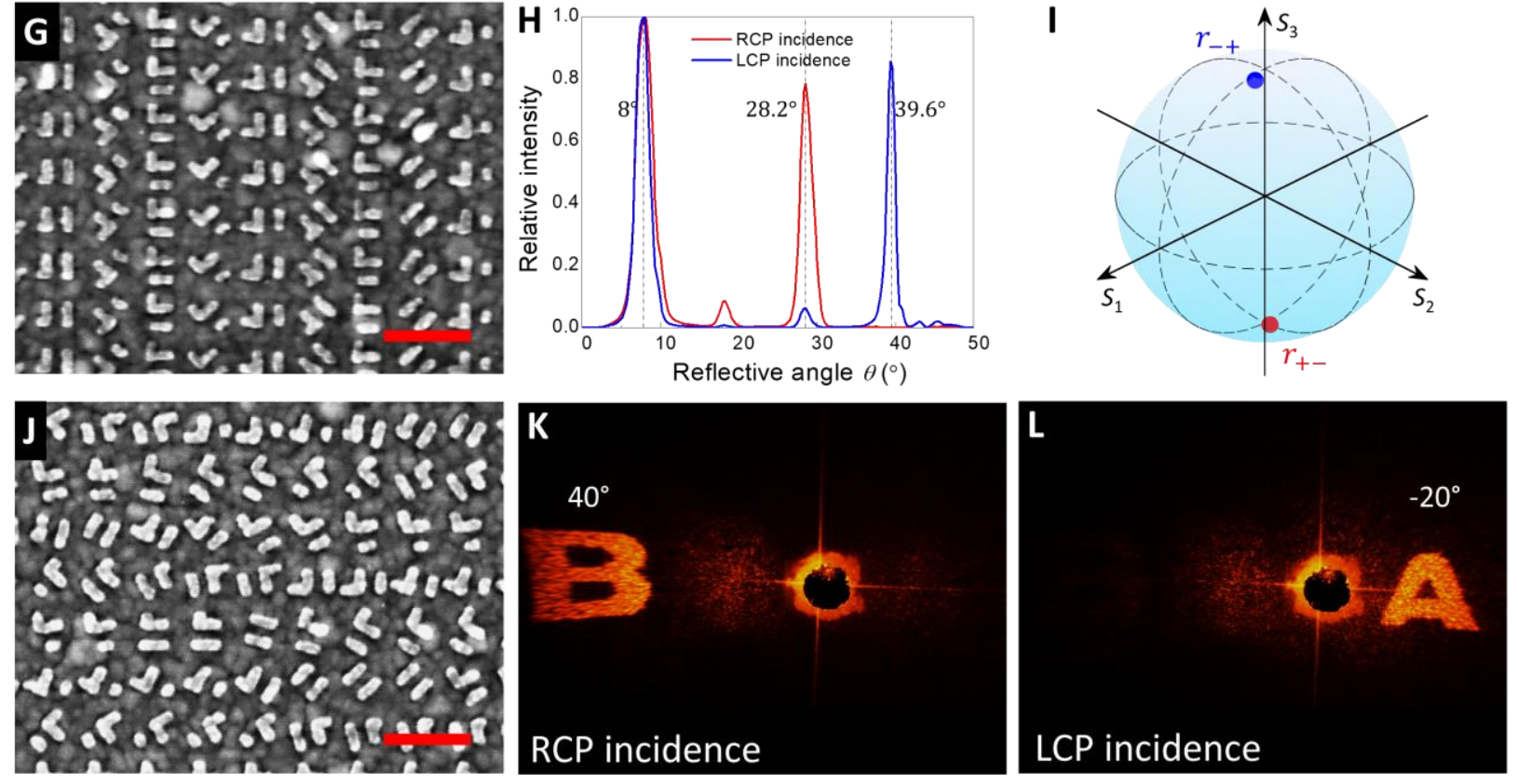

Fig. 4. Experimental results of the topological metasurface. (A) Fabricated nanostructures for the ET phase meta-deflector. (B) Measured far field power patterns for RCP (red solid curves) and LCP (blue dot curves) incidence on ET phase meta-deflector. The phase gradient between adjacent meta-atoms is designed as $90^{\circ}$. The first order of the CP converted beam $r_{+-}$is deflected from $34^{\circ}$ to $\sim 44^{\circ}$ when the wavelength $\lambda$ varies from $500 \mathrm{~nm}$ to $\sim 675 \mathrm{~nm}$, while the other CP converted beam of $r_{-+}$is undeflected (at the zero order of $8^{\circ}$ ). (C) Measured efficiency of the deflector (red dots) and measured power ratio of $\left|r_{+-}\right| /\left|r_{-+}\right|$at the deflected angle (blue stars). (D) Fabricated nanostructures for an ET phase meta-hologram designed by encoding the holographic phase profile 
into the deflector. (E) A holographic image of "C" for $r_{+-}$is displayed at the angle of $30^{\circ}$, (F) but no image appears for $r_{-+}$. (G) Fabricated nanostructures for an ET+PB phase meta-deflector. $(\mathrm{H})$ Measured far field power patterns with RCP (red curve) and LCP (blue curve) incidence on ET+PB phase meta-deflector. The phase gradient between adjacent meta-atoms is designed as $60^{\circ}$ and $90^{\circ}$ respectively for $r_{+-}$and $r_{-+}$. (I) Poincaré sphere representation of the measured polarization of $r_{+-}$(red dot) and $r_{-+}$(blue dot) at the first deflected order. (J) Fabricated nanostructures for an $\mathrm{ET}+\mathrm{PB}$ phase meta-hologram. (K) Holographic images of "B" for $r_{+-}$and (L) "A" for $r_{-+}$are displayed at the designed angle of $40^{\circ}$ and $-20^{\circ}$, respectively. All the curves in $\mathrm{B}$ and $\mathrm{H}$ are normalized with the zero order as unity. The operating wavelength in $\mathrm{E}, \mathrm{F}, \mathrm{H}, \mathrm{K}$ and $\mathrm{L}$ is $\lambda=$ $600 \mathrm{~nm}$. The incident angles of the deflectors and holograms are $8^{\circ}$ and $0^{\circ}$, respectively. The scale bar in $\mathrm{A}, \mathrm{D}, \mathrm{G}$, and $\mathrm{J}$ corresponds to $500 \mathrm{~nm}$. 


\section{Science $\triangle 1$ AAAS}

\section{Supplementary Materials for}

\section{Plasmonic Topological Metasurface by Encircling an Exceptional Point}

Authors: Qinghua Song ${ }^{1}$, Mutasem Odeh ${ }^{2}$, Jesús Zúñiga-Pérez ${ }^{1}$, Boubacar Kanté2 ${ }^{2}$ and Patrice Genevet $^{1 *}$

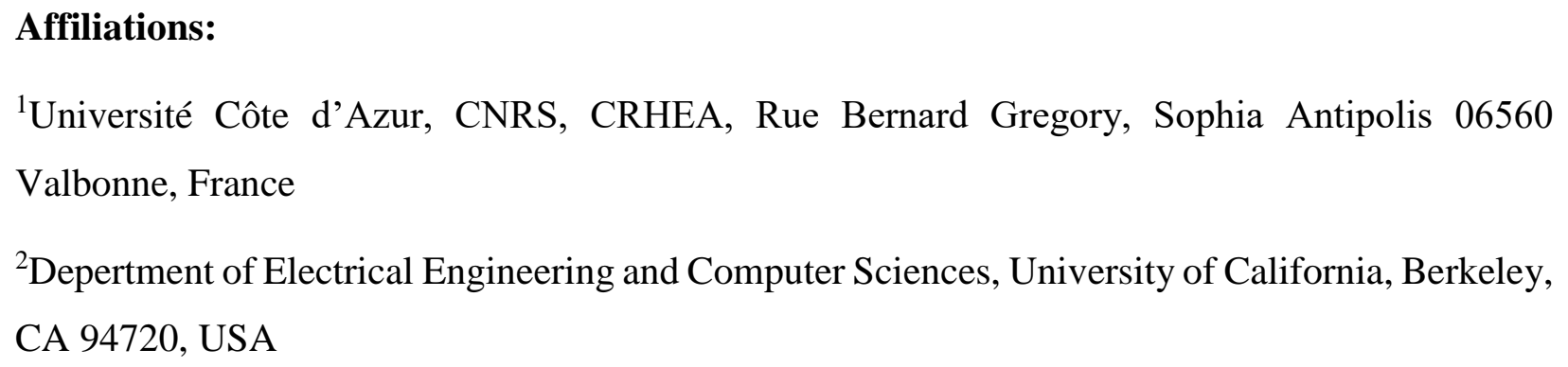

${ }^{1}$ Université Côte d'Azur, CNRS, CRHEA, Rue Bernard Gregory, Sophia Antipolis 06560 Valbonne, France

${ }^{2}$ Depertment of Electrical Engineering and Computer Sciences, University of California, Berkeley, CA 94720, USA

15

*Correspondence to: Patrice.Genevet@ crhea.cnrs.fr.

20

This PDF file includes:

Materials and Methods

Figs. S1 to S14

Notes 1-5 


\section{Materials and Methods}

The fabrication processes and the measurement setup are shown in Fig. S1 and S2, respectively.

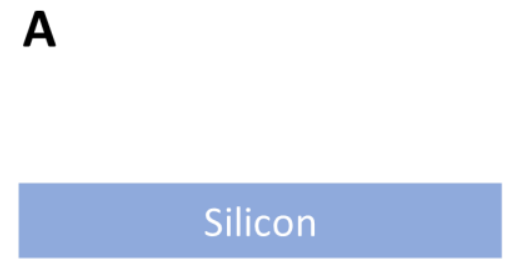

Substrate

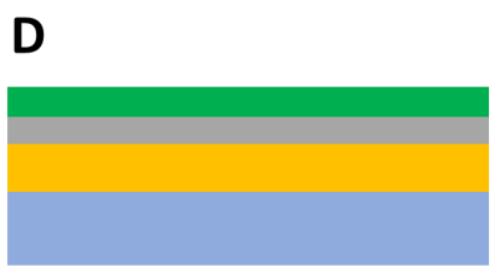

Spin coating resist

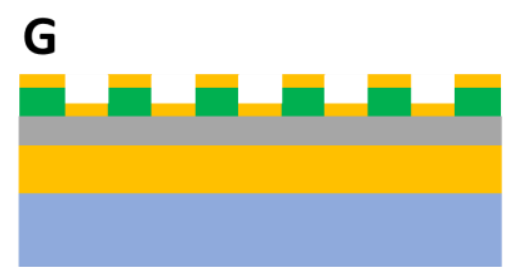

Al deposition

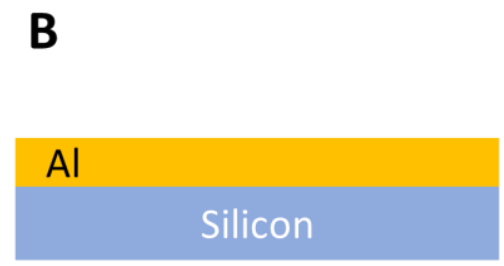

Al deposition

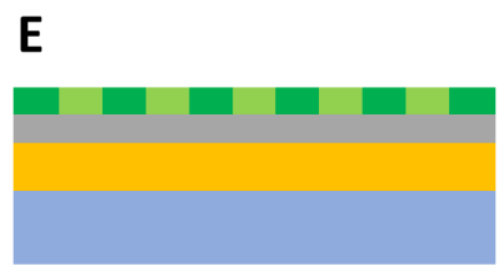

EBL

H

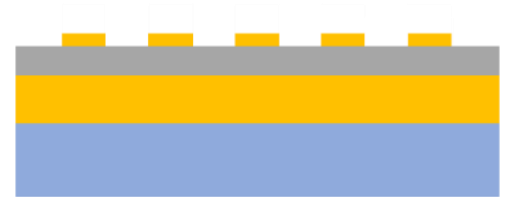

Liftoff

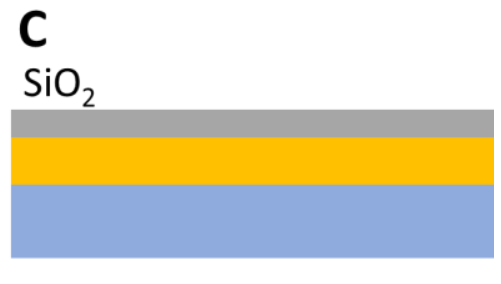

$\mathrm{SiO}_{2}$ deposition

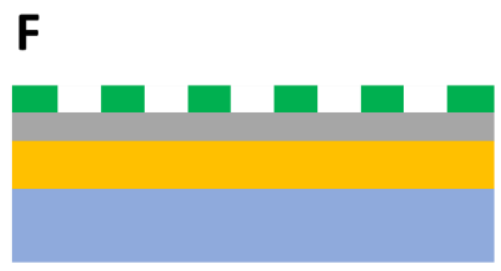

Development

Fig. S1. Plasmonic metasurface fabrication process. (A) Cleaning and dicing a silicon wafer into a $2 \times 2 \mathrm{~cm}$ samples. (B) E-beam evaporation of $150 \mathrm{~nm}$ ground Al layer. (C) PECVD deposition of $40 \mathrm{~nm} \mathrm{SiO} 2$ spacer layer. (D) Spin-coat and softback $220 \mathrm{~nm}$ PMMA-A4 e-beam resist. (E) $100 \mathrm{keV}$ e-beam lithography exposure of shape-corrected structure using BEAMERGenISys software. (F) Cold-development using MBIK/IPA 1:3 for 2 minutes, followed by O2plasma descumming process for 10 seconds. (G) E-beam evaporation of $30 \mathrm{~nm}$ Al device layer, and $(\mathrm{H})$ lift-off process using room-temperature acetone solvent followed by 10 -seconds sonication bath. 


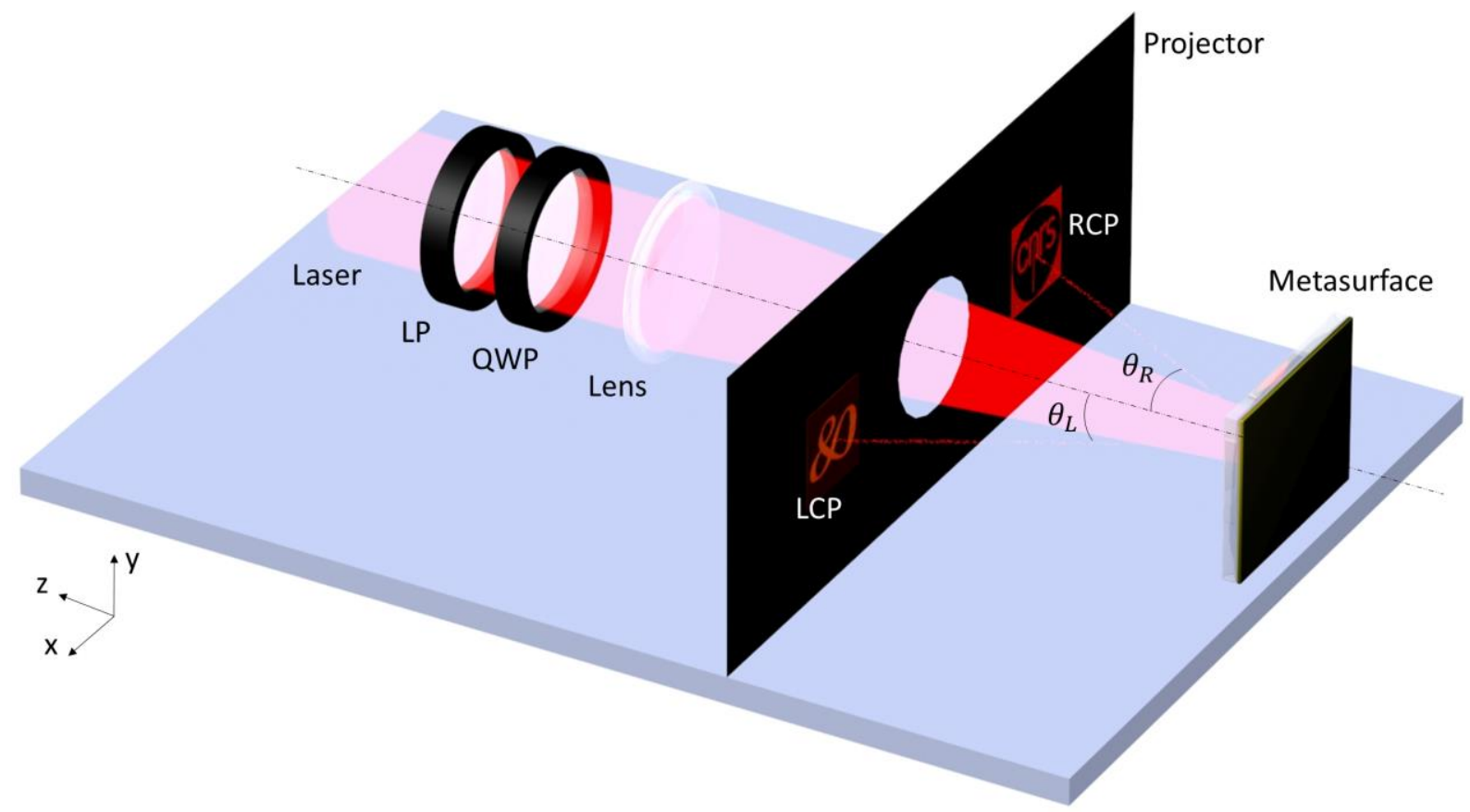

Fig. S2. Optical setup for the measurement of the meta-hologram. A laser beam at visible range propagates through a linear polarizer and quarter wavelength, so that a circular polarized light is obtained. Then the laser beam passes through a lens to weakly focus on the meta-hologram. The reflected holographic images are projected onto a projector with a hole in the center for the incident light passing through. LP: linear polarizer; QWP: quarter wave plate. 


\section{Supplementary Note 1: Phase accumulation by encircling a singularity point.}

Consider an arbitrary complex number as a function of parameter $\boldsymbol{R}$,

$$
f(\boldsymbol{R})=\operatorname{Re}[f(\boldsymbol{R})]+i \operatorname{Im}[f(\boldsymbol{R})]
$$

where $\operatorname{Re}[f(\boldsymbol{R})]$ and $\operatorname{Im}[f(\boldsymbol{R})]$ represent the real and imaginary part of $f(\boldsymbol{R})$, respectively, $i^{2}=$ -1 . The phase of complex number $f(\boldsymbol{R})$ can be described as

$$
\phi[f(\boldsymbol{R})]=\angle f(\boldsymbol{R})=\operatorname{atan} \frac{\operatorname{Im}[f(\boldsymbol{R})]}{\operatorname{Re}[f(\boldsymbol{R})]}
$$

When the parameter $\boldsymbol{R}$ varies continuously from point $\boldsymbol{P}_{\mathbf{1}}$ to $\boldsymbol{P}_{\mathbf{2}}$, the accumulated phase $\Phi$ along the path $\boldsymbol{P}_{\mathbf{1}} \rightarrow \boldsymbol{P}_{\mathbf{2}}$ in the complex plane $(\operatorname{Re}[f(\boldsymbol{R})], \operatorname{Im}[f(\boldsymbol{R})])$ as shown in fig. S3A is given by

$$
\Phi=\int_{\phi\left[f\left(\boldsymbol{P}_{1}\right)\right]}^{\phi\left[f\left(\boldsymbol{P}_{2}\right)\right]} d \phi
$$

For any closed path $l$, if the origin is within the enclosed path $l_{1}$ as shown in fig. S3B, the winding number (28) of path $l_{1}$ around the origin $w=\frac{1}{2 \pi} \oint d \phi=1$. Therefore, the accumulated phase along the path $l_{1}$ is

$$
\Phi=\oint d \phi=2 \pi
$$

However, if the origin is outside the enclosed path $l_{2}$ as shown in fig. $\mathrm{S} 3 \mathrm{C}$, the winding number is 0 . Therefore, the accumulated phase along the path $l_{2}$ is

$$
\Phi=\oint d \phi \neq 2 \pi
$$

Assume that for a parameter space of $\boldsymbol{R}$, the complex number $f(\boldsymbol{R})$ vanishes at the parameter of $\boldsymbol{P}^{s}$, i.e., $f\left(\boldsymbol{P}^{s}\right)=\operatorname{Re}\left[f\left(\boldsymbol{P}^{s}\right)\right]+i \operatorname{Im}\left[f\left(\boldsymbol{P}^{s}\right)\right]=0$. We will refer to such point of $\boldsymbol{P}^{s}\left(f\left(\boldsymbol{P}^{s}\right)=\right.$ 0 ) as singularity point $\left(\boldsymbol{P}^{\boldsymbol{s}}\right)$ in this paper. For clarification, in the following text the variable $\boldsymbol{R}$ is defined in a two-dimensional parameter space $\left(\boldsymbol{R}_{1}, \boldsymbol{R}_{2}\right)$, the complex number $f(\boldsymbol{R})$ is defined in the two-dimensional complex plane of $(\operatorname{Re}[f(\boldsymbol{R})], \operatorname{Im}[f(\boldsymbol{R})])$. When the parameter $\boldsymbol{R}$ is varied along a closed path in the parameter space of $\left(\boldsymbol{R}_{\mathbf{1}}, \boldsymbol{R}_{\mathbf{2}}\right)$ as shown in fig. S4A and crosses the singularity point $\boldsymbol{P}^{s}$, the complex number $f(\boldsymbol{R})$ will pass through the origin in the complex plane $(\operatorname{Re}[f(\boldsymbol{R})], \operatorname{Im}[f(\boldsymbol{R})])$. Therefore, at least $\pi$ phase can be accumulated as shown in fig. S4B. 
Assume that at the vicinity of the singularity point $\boldsymbol{P}^{\boldsymbol{s}}$, the parameter $\boldsymbol{R}$ deviates from the previous path and encircles the singularity point $\boldsymbol{P}^{\boldsymbol{s}}$, as shown in fig. S4C. The complex number $f(\boldsymbol{R})$ will no longer pass through the origin in the complex plane of $(\operatorname{Re}[f(\boldsymbol{R})], \operatorname{Im}[f(\boldsymbol{R})])$. There are two possibilities for the evolution of the function $f(\boldsymbol{R})$, as shown in fig. S4D. Either it will encircle the origin, as shown by the red solid path (combined with the blue path), so that a full $2 \pi$ phase can be accumulated, or the origin will be outside of the closed path as shown by the red dash path (combined with blue path), such that the accumulated phase is not $2 \pi$.
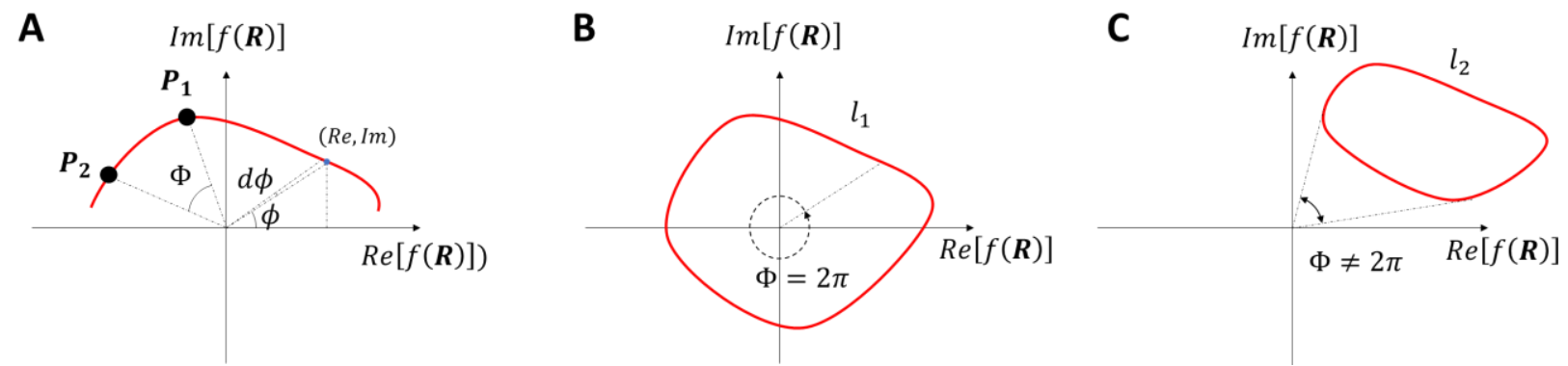

Fig. S3. Phase accumulation along a closed path in any parameter space $\boldsymbol{R}$. (A) $R e[f(\boldsymbol{R})]$ and $\operatorname{Im}[f(\boldsymbol{R})]$ represent the real and imaginary part of function $f(\boldsymbol{R})$, which are related to the parameter $\boldsymbol{R}$. The phase of $f(\boldsymbol{R})$ is represented by $\phi$. The phase accumulation along the path $\boldsymbol{P}_{\mathbf{1}} \rightarrow$ $\boldsymbol{P}_{\mathbf{2}}$ is $\Phi$. (B) When the singularity point of $f\left(\boldsymbol{P}^{\boldsymbol{s}}\right)=0$, i.e., the origin of the complex plane, is within the enclosed path $l_{1}$, a full $2 \pi$ phase is accumulated along the path. (C) When the singularity point of $f\left(\boldsymbol{P}^{s}\right)=0$, i.e., the origin of the complex plane, is outside of the enclosed path $l_{2}$, the accumulated phase along the path is no longer $2 \pi$. The winding number of paths $l_{1}$ and $l_{2}$ along the origin is 1 and 0 , respectively. 

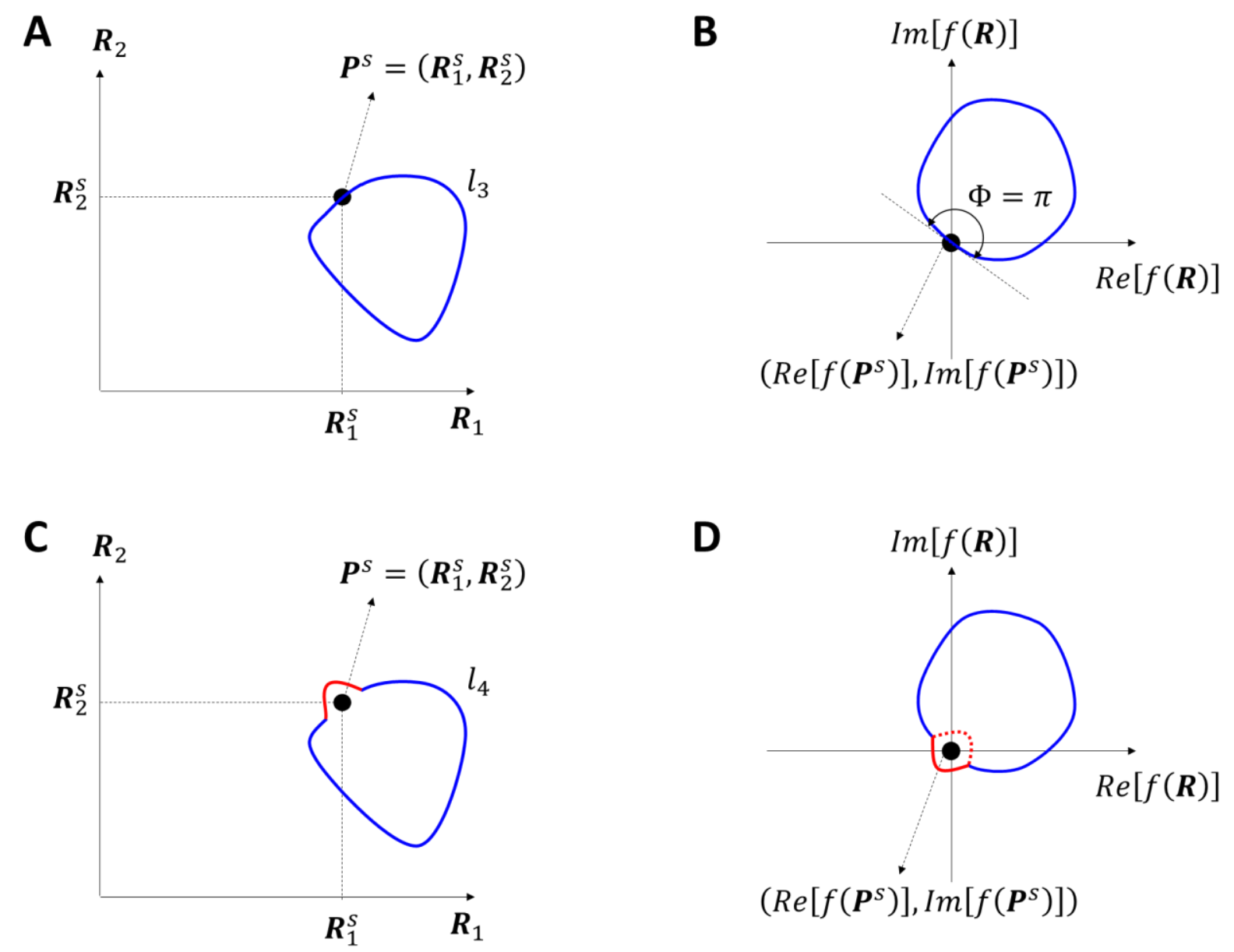

Fig. S4. Phase accumulation along a closed path in a two-dimensional parameter space $R=$ $\left(\boldsymbol{R}_{\mathbf{1}}, \boldsymbol{R}_{\mathbf{2}}\right)$. (A) The singularity point $f\left(\boldsymbol{P}^{s}\right)=0$ exists at the parameter of $\boldsymbol{P}^{s}=\left(\boldsymbol{R}_{\mathbf{1}}^{s}, \boldsymbol{R}_{\mathbf{2}}^{s}\right)$. The blue closed path $l_{3}$ crosses the singularity point. (B) The corresponding function $f(\boldsymbol{R})=\operatorname{Re}[f(\boldsymbol{R})]+$ $i \operatorname{Im}[f(\boldsymbol{R})]$ crosses the origin in the complex plane. (C) At the vicinity of the singularity point, the parameter $\left(\boldsymbol{R}_{\mathbf{1}}, \boldsymbol{R}_{\mathbf{2}}\right)$ deviates from the singularity point as shown in the red curve and encircles the singularity point by combining red and blue path $l_{4}$. (D) Two possibilities of evolution of the function $f(\boldsymbol{R})$ can be observed. The origin in the complex plane can be either within (red solid path) or out of (red dash path) the closed path of $f(\boldsymbol{R})$. For the red solid path together with blue path, a full $2 \pi$ phase can be realized. For the red dash path together with the blue path, the phase accumulation is no longer $2 \pi$. 


\section{Supplementary Note 2: Robustness of the $2 \pi$ phase accumulation: topological protection.}

Here we consider the case of a closed path of $\boldsymbol{R}=\left(\boldsymbol{R}_{\mathbf{1}}, \boldsymbol{R}_{\mathbf{2}}\right)$ that encircles the singularity point $\boldsymbol{P}^{\boldsymbol{s}}=\left(\boldsymbol{R}_{\mathbf{1}}^{\boldsymbol{s}}, \boldsymbol{R}_{\mathbf{2}}^{\boldsymbol{S}}\right)$, which implies that the closed path of $f(\boldsymbol{R})$ in the complex plane encircles the origin, as shown in the blue closed path of fig. S5A and S5B. The accumulated phase of $f(\boldsymbol{R})$ when encircling the singularity point $\boldsymbol{P}^{\boldsymbol{s}}$ is $2 \pi$ according to Eq. S4 as discussed in Note 1. Assume that the blue closed path is changed continuously to the red closed path without crossing the singularity point $\boldsymbol{P}^{s}$ as shown in fig. S5A. If the origin jumps outside of the function $f(\boldsymbol{R})$ as shown in fig. S5D, then there must exist an intermediate yellow path in parameter space $\left(\boldsymbol{R}_{\mathbf{1}}, \boldsymbol{R}_{\mathbf{2}}\right)$ that imposes $f(\boldsymbol{R})$ to pass through the origin, as shown in fig. S5C. In this case, there must exist an additional singularity point of $\boldsymbol{P}^{\boldsymbol{s}}$, as shown by the yellow dot in fig. S5A, such that $f\left(\boldsymbol{P}^{\boldsymbol{s}}\right)=$ $\operatorname{Re}\left[f\left(\boldsymbol{P}^{\boldsymbol{s}^{\prime}}\right)\right]+i \operatorname{Im}\left[f\left(\boldsymbol{P}^{\boldsymbol{s}^{\prime}}\right)\right]=0$, as shown by the yellow dot in fig. S5C. Therefore, there must exist at least two singularity points $\boldsymbol{P}^{\boldsymbol{s}, \boldsymbol{s} \mathbf{}}$ such that the winding number of function $f(\boldsymbol{R})$ along the origin changes from 1 to 0 (from blue state in fig. S5B to red state in fig. S5D). In other words, a robust $2 \pi$ phase accumulation is topologically protected by this singularity point unless the topology is broken by introducing another singularity point. In conclusion, if the following two conditions are satisfied a $2 \pi$ phase accumulation is guaranteed for any closed path that encircles the singularity at point $\boldsymbol{P}^{\boldsymbol{s}}$ in parameter space: (1) there exists only one singularity point $\boldsymbol{P}^{\boldsymbol{s}}$ such that $f\left(\boldsymbol{P}^{\boldsymbol{s}}\right)=0$, (2) there exists one enclosed path that encircles the singularity point $\boldsymbol{P}^{\boldsymbol{s}}$ such that the origin is within the path of $f(\boldsymbol{R})$ in the complex plane. 

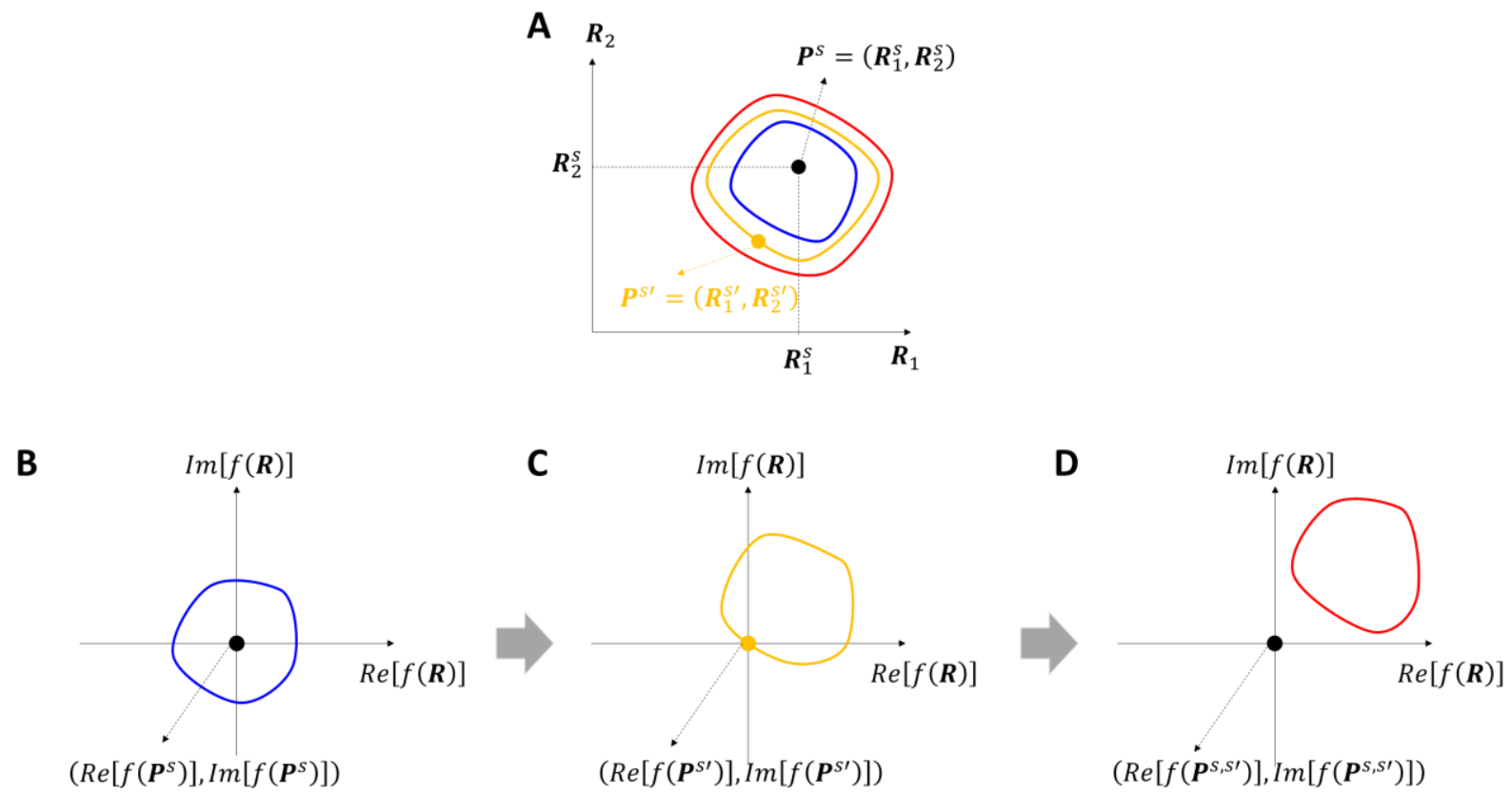

Fig. S5. Topology protection of $2 \pi$ phase. (A) Three closed paths in the parameter space $\boldsymbol{R}=$ $\left(\boldsymbol{R}_{1}, \boldsymbol{R}_{2}\right)$. The singularity point exists at the parameter of $\boldsymbol{P}^{s}=\left(\boldsymbol{R}_{1}^{s}, \boldsymbol{R}_{2}^{s}\right)$. The parameter is continuously changed from blue to yellow, and then to red path. All the three paths are encircling the singularity point of $\boldsymbol{P}^{\boldsymbol{s}}$. The corresponding path of $f(\boldsymbol{R})$ in the complex plane is shown in (B) blue, (C) yellow and (D) red paths. Assume the blue path of $f(\boldsymbol{R})$ is encircling the origin, i.e., a full $2 \pi$ phase is accumulated along the closed path. When the path of $f(\boldsymbol{R})$ is changed from (B) to (D), there must exist an intermediate path as shown in (C), such that the function $f(\boldsymbol{R})$ passes through the origin. In other words, there must exist another singularity point of $\boldsymbol{P}^{\boldsymbol{s}^{\prime}}=\left(\boldsymbol{R}_{1}^{\boldsymbol{s}^{\prime}}, \boldsymbol{R}_{2}^{\boldsymbol{s}^{\prime}}\right)$ in the yellow path in (A). Therefore, the full $2 \pi$ phase is topologically protected by $\boldsymbol{R}^{\boldsymbol{s}}$. By introducing another singularity point, the topology protection of $2 \pi$ phase is broken. 


\section{Supplementary Note 3: General mathematic study of a planar chiral non-Hermitian matrix.}

In this paper we only consider the metasurface with planar chiral anisotropic properties in the reflection regime, i.e.,

$$
\left\{\begin{array}{l}
r_{x x} \neq r_{y y} \\
r_{x y}=r_{y x}
\end{array}\right.
$$

where $r_{j k}$ is the reflection from $k$ polarized input light to $j$ polarized output light. $r_{x x} \neq r_{y y}$ due to the anisotropy, and $r_{x y}=r_{y x}$ due to the reciprocity. The reflection matrix can be described as,

$$
\hat{r}(\boldsymbol{R})=\left(\begin{array}{ll}
r_{x x}(\boldsymbol{R}) & r_{x y}(\boldsymbol{R}) \\
r_{y x}(\boldsymbol{R}) & r_{y y}(\boldsymbol{R})
\end{array}\right)=\left(\begin{array}{cc}
a(\boldsymbol{R})+i b(\boldsymbol{R}) & c(\boldsymbol{R})+i d(\boldsymbol{R}) \\
c(\boldsymbol{R})+i d(\boldsymbol{R}) & e(\boldsymbol{R})+i f(\boldsymbol{R})
\end{array}\right)
$$

where $a, b, c, d, e, f$ are arbitrary real values that are related to arbitrary system parameter $\boldsymbol{R}$. For simplicity, in some of the following discussions, we ignore the notation of $(\boldsymbol{R})$. Assume the eigenvalues and eigenstates of the matrix $\hat{r}$ is $\lambda_{1,2}$ and $\left|n_{1,2}\right\rangle$, i.e.,

$$
\hat{r}\left|n_{1,2}\right\rangle=\lambda_{1,2}\left|n_{1,2}\right\rangle
$$

The eigenvalues can be derived as,

$$
\lambda_{1,2}=\frac{(a+i b)+(e+i f) \pm \sqrt{[(a+i b)-(e+i f)]^{2}+4(c+i d)^{2}}}{2}
$$

At the exceptional point of $\boldsymbol{P}^{e}$, the eigenvalues degenerate, i.e., the square root of $\lambda_{1,2}$ vanishes as,

$$
\begin{aligned}
& \sqrt{\left[\left(a\left(\boldsymbol{P}^{e}\right)+i b\left(\boldsymbol{P}^{e}\right)\right)-\left(e\left(\boldsymbol{P}^{e}\right)+i f\left(\boldsymbol{P}^{e}\right)\right)\right]^{2}+4\left(c\left(\boldsymbol{P}^{e}\right)+i d\left(\boldsymbol{P}^{e}\right)\right)^{2}} \\
= & \sqrt{[(a+i b)-(e+i f)-2 i(c+i d)][(a+i b)-(e+i f)+2 i(c+i d)]} \\
= & \sqrt{[(a-e+2 d)+i(b-f-2 c)][(a-e-2 d)+i(b-f+2 c)]} \\
= & 0
\end{aligned}
$$

We then get

$$
\left\{\begin{array}{l}
a\left(\boldsymbol{P}^{e}\right)-e\left(\boldsymbol{P}^{e}\right)+2 d\left(\boldsymbol{P}^{e}\right)=0 \\
b\left(\boldsymbol{P}^{e}\right)-f\left(\boldsymbol{P}^{e}\right)-2 c\left(\boldsymbol{P}^{e}\right)=0
\end{array}\right.
$$

or 


$$
\left\{\begin{array}{l}
a\left(\boldsymbol{P}^{e^{\prime}}\right)-e\left(\boldsymbol{P}^{e^{\prime}}\right)-2 d\left(\boldsymbol{P}^{e^{\prime}}\right)=0 \\
b\left(\boldsymbol{P}^{e^{\prime}}\right)-f\left(\boldsymbol{P}^{e^{\prime}}\right)+2 c\left(\boldsymbol{P}^{e^{\prime}}\right)=0
\end{array}\right.
$$

If the reflection matrix $\hat{r}(\boldsymbol{R})$ is Hermitian, i.e., $b(\boldsymbol{R})=d(\boldsymbol{R})=f(\boldsymbol{R})=0$, Eq. S11 and S12 coalesce as $a\left(\boldsymbol{P}^{e}\right)=e\left(\boldsymbol{P}^{e}\right)$, and $c\left(\boldsymbol{P}^{e}\right)=0$. The eigenstates are $\left|n_{1}\right\rangle=\left(\begin{array}{l}1 \\ 0\end{array}\right),\left|n_{1}\right\rangle=\left(\begin{array}{l}0 \\ 1\end{array}\right)$, which correspond to linearly-polarized orthogonal eigenstates.

However, if the reflection matrix $\hat{r}(\boldsymbol{R})$ is non-Hermitian, i.e., one of the imaginary parts $b, d, f$ does not vanish, Eq. S11 and S12 give rise to two different cases. Consider the case of Eq. S11, i.e., $(a-e+2 d)+i(b-f-2 c)=0$. According to Eq. S7-9 and S11, the degenerate eigenstates can be calculated as

$$
\left|n_{1}\right\rangle=\left|n_{2}\right\rangle=|-\rangle=\left(\begin{array}{c}
1 \\
-i
\end{array}\right)
$$

which correspond to right circular polarization $(|-\rangle)$, i.e., $\hat{r}\left(\boldsymbol{P}^{e}\right)|-\rangle=\lambda_{1,2}\left(\boldsymbol{P}^{e}\right)|-\rangle$. That means that when the incident light is pure RCP, the output light is also pure RCP, i.e., the CP conversion channel from RCP to LCP is blocked as,

$$
r_{+-}\left(\boldsymbol{P}^{e}\right)=0
$$

where the subscript + and - represent LCP and RCP, respectively.

Since in the main text we will focus on experiments based on circularly-polarized beams, we will rewrite the matrix $\hat{r}$ in a circular polarization basis,

$$
\hat{r}=\left(\begin{array}{ll}
r_{++} & r_{+-} \\
r_{-+} & r_{--}
\end{array}\right)=\frac{1}{2}\left(\begin{array}{ll}
r_{x x}+r_{y y}+i\left(r_{x y}-r_{y x}\right) & r_{x x}-r_{y y}-i\left(r_{x y}+r_{y x}\right) \\
r_{x x}-r_{y y}+i\left(r_{x y}+r_{y x}\right) & r_{x x}+r_{y y}-i\left(r_{x y}-r_{y x}\right)
\end{array}\right)
$$

If the reflection matrix $\hat{r}(\boldsymbol{R})$ is Hermitian, $r_{+-}=a-e-i 2 c$, and $r_{-+}=a-e+i 2 c$, the amplitude of $r_{+-}$always equals to $r_{-+}$(see fig. S6A). However, for Non-Hermitian reflection matrix $\hat{r}(\boldsymbol{R}), r_{+-}=\frac{1}{2}(a-e+2 d)+\frac{i}{2}(b-f-2 c)$, and $r_{-+}=\frac{1}{2}(a-e-2 d)+\frac{i}{2}(b-f+$ $2 c$ ), we get unequal reflection for the two circular polarizations, i.e., $\left|r_{+-}\right| \neq\left|r_{-+}\right|$(see fig. S6C). Consider the circular polarization conversion term $r_{+-}$and note that it is related to the system parameter $\boldsymbol{R}$ as,

$$
\begin{aligned}
r_{+-}(\boldsymbol{R})=r_{x x}(\boldsymbol{R})-r_{y y}(\boldsymbol{R})-i\left(r_{x y}(\boldsymbol{R})+r_{y x}(\boldsymbol{R})\right) \\
=\frac{1}{2}((a(\boldsymbol{R})-e(\boldsymbol{R})+2 d(\boldsymbol{R}))+i(b(\boldsymbol{R})-f(\boldsymbol{R})-2 c(\boldsymbol{R}))) \\
=\operatorname{Re}\left[r_{+-}(\boldsymbol{R})\right]+i \operatorname{Im}\left[r_{+-}(\boldsymbol{R})\right]
\end{aligned}
$$


According to Eq. S11, we get that at the exceptional point $\boldsymbol{P}^{e}$,

$$
\left\{\begin{array}{l}
\operatorname{Re}\left[r_{+-}\left(\boldsymbol{P}^{e}\right)\right]=a\left(\boldsymbol{P}^{e}\right)-e\left(\boldsymbol{P}^{e}\right)+2 d\left(\boldsymbol{P}^{e}\right)=0 \\
\operatorname{Im}\left[r_{+-}\left(\boldsymbol{P}^{e}\right)\right]=b\left(\boldsymbol{P}^{e}\right)-f\left(\boldsymbol{P}^{e}\right)-2 c\left(\boldsymbol{P}^{e}\right)=0
\end{array}\right.
$$

which is consistent with the analysis of Eq. S14. Therefore, the exceptional point $\boldsymbol{P}^{e}$ is a singularity point $\left(\boldsymbol{P}^{S}\right.$ as discussed in Note $\left.1-2\right)$ for $r_{+-}(\boldsymbol{R})$.

Similarly, if we consider the case of Eq. S12, the eigenstates degenerate as LCP $(|+\rangle)$ at the exceptional point $\boldsymbol{P}^{e \prime}$,

$$
\left|n_{1}\right\rangle=\left|n_{2}\right\rangle=|+\rangle=\left(\begin{array}{l}
1 \\
i
\end{array}\right)
$$

We have,

$$
r_{-+}\left(\boldsymbol{P}^{e \prime}\right)=0
$$

Therefore, the exceptional point $\boldsymbol{P}^{e \prime}$ is a singularity point $\left(\boldsymbol{P}^{s}\right.$ as discussed in Note 1-2) for $r_{-+}(\boldsymbol{R})$. 

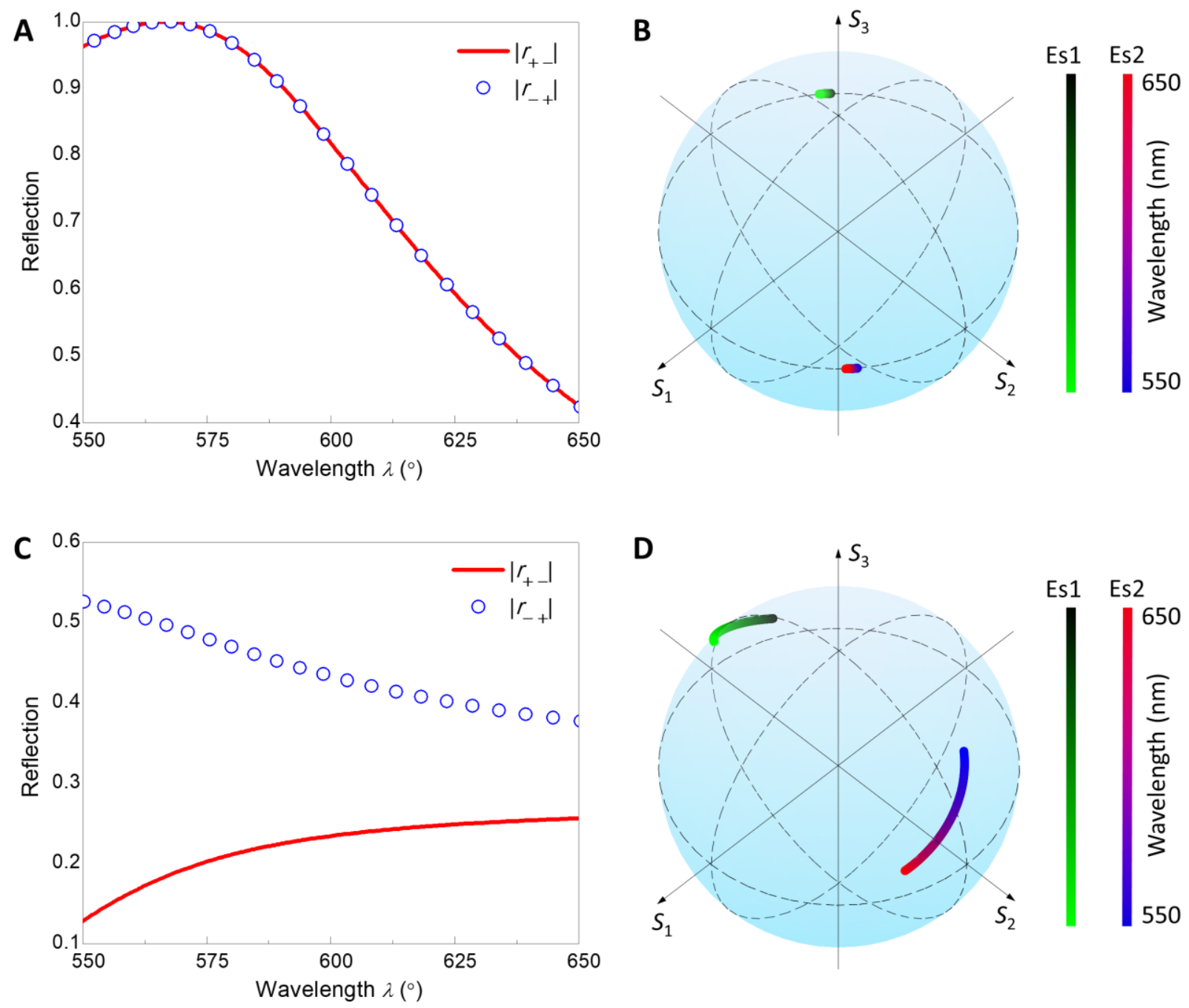

Fig. S6. Comparison of lossless and lossy 2D chiral metasurfaces. (A) Simulated reflective CP conversion and (B) the corresponding eigenstates with perfect electrical conductor (PEC) structure. The amplitude of the two converted CP beams is the same. The two eigenstates are always linear polarized (on the equator of the Poincaré sphere). (C) Simulated reflective CP conversion and (D) the corresponding eigenstates with Al structure. The amplitude of the two converted CP beams becomes different. The two eigenstates become elliptical polarized with the same handedness (on the top sphere cap of the Poincaré sphere). The dimension of the structure in this case is $L_{1}=50 \mathrm{~nm}, L_{2}=140 \mathrm{~nm}, L_{3}=100 \mathrm{~nm}, P=300 \mathrm{~nm}, w=40 \mathrm{~nm}, g=50 \mathrm{~nm}, h=25 \mathrm{~nm}$, $h_{1}=40 \mathrm{~nm}, h_{2}=150 \mathrm{~nm}$. See more details of the discussion in Supplementary Note 3 . 
A

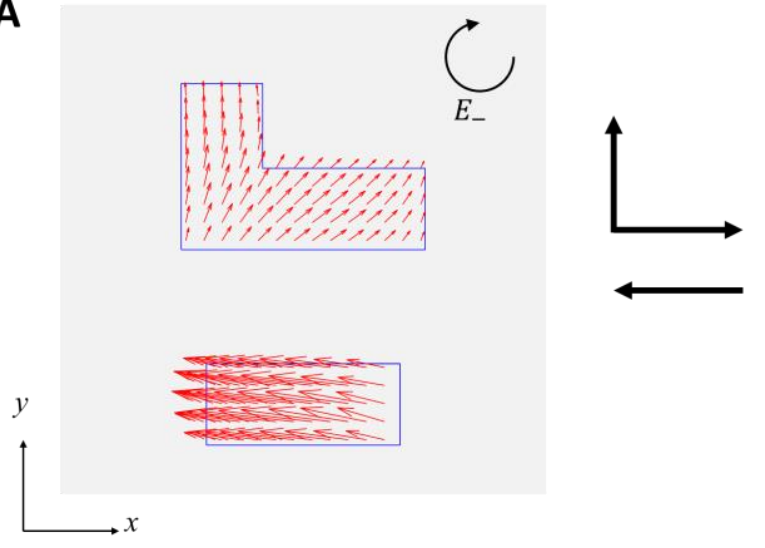

B

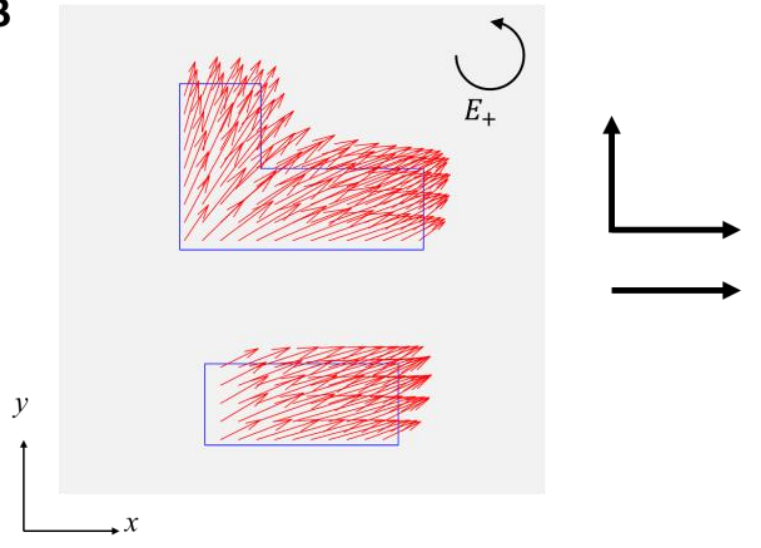

Fig. S7. Simulated surface current distribution at the exceptional point with (A) RCP and (B) LCP incidence. The two horizontal rods have bonding and anti-bonding current distribution under the two CP beam incidences. Changing the geometrical parameters of the meta-structure leads to destructive interference condition between the polarization converted from RCP to LCP and the unconverted fields of the reflected LCP, resulting in a zero reflection of $r_{+-}$, but leaving a non-zero reflection of $r_{-+}$due to the different response of the surface current. 


\section{Supplementary Note 4: Coupled-mode theory.}

The metasurface consists of a "느" shape nanostructure stacked with an L shape rod and a straight rod in each meta-atom (see Fig. 1A in the main text). The structure can be modeled using coupled-mode theory inspired from the references of (19-20). The effective dipole moment of the structure is $p_{x, y}=\tilde{p}_{x, y} e^{i \omega t}$, which couples strongly to an incident radiation field $E_{i}=\tilde{E}_{i} e^{i \omega t}$ with a radiative coupling strength $g_{j}$. The resonant frequency and damping coefficient are $\omega_{0}$ and $\gamma$, respectively. Assume the incident radiation is close to the resonance $\left(\delta=\omega-\omega_{0} \approx 0\right)$ and damping is small $\left(\gamma \ll \omega_{0}\right)$, the dipole moments of the nanostructure in the coupled system are related to the incident electric fields through the polarizability matrix as,

$$
\left[\left(\begin{array}{cc}
\delta_{x}+i \gamma_{x} & 0 \\
0 & \delta_{y}+i \gamma_{y}
\end{array}\right)+\left(\begin{array}{cc}
G_{x x} & G_{x y} \\
G_{y x} & G_{y y}
\end{array}\right)\right]\left(\begin{array}{l}
p_{x} \\
p_{y}
\end{array}\right)=\left(\begin{array}{l}
g_{x} E_{i x} \\
g_{y} E_{i y}
\end{array}\right)
$$

where the coupling term $G_{j k}$ is a summation of the $j$-oriented radiation fields formed by the $k$ oriented dipole moments, which satisfies the following condition $G_{x x}=G_{y y}$ due to the square lattice and $G_{x y}=G_{y x}$ due to the reciprocity. Therefore, the dipole moments can be retrieved as

$$
\begin{aligned}
& \left(\begin{array}{l}
p_{x} \\
p_{y}
\end{array}\right)=\left(\begin{array}{cc}
\delta_{x}+i \gamma_{x}+G_{x x} & G_{x y} \\
G_{x y} & \delta_{y}+i \gamma_{y}+G_{x x}
\end{array}\right)^{-1}\left(\begin{array}{l}
g_{x} E_{i x} \\
g_{y} E_{i y}
\end{array}\right) \\
& =\frac{1}{\operatorname{det} A}\left(\begin{array}{cc}
\delta_{y}+i \gamma_{y}+G_{x x} & -G_{x y} \\
-G_{x y} & \delta_{x}+i \gamma_{x}+G_{x x}
\end{array}\right)\left(\begin{array}{l}
g_{x} E_{i x} \\
g_{y} E_{i y}
\end{array}\right)
\end{aligned}
$$

where $\operatorname{det} A$ is the determinant of matrix $A=\left(\begin{array}{cc}\delta_{x}+i \gamma_{x}+G_{x x} & G_{x y} \\ G_{x y} & \delta_{y}+i \gamma_{y}+G_{x x}\end{array}\right)$. The reflective field can be obtained by superposing the incident field reflected back from the ground mirror and the field radiated in the forward direction due to the oscillating dipoles, given by $\frac{i \omega \eta_{0}}{2 a^{2}}\left(g_{x} p_{x}, g_{y} p_{y}\right)$, where $\eta_{0}=\sqrt{\mu_{0} / \varepsilon_{0}}$ is the free space impedance and $a$ is the lattice constant. Therefore, the reflective field is,

$$
\begin{gathered}
\left(\begin{array}{c}
E_{o x} \\
E_{o y}
\end{array}\right)=\hat{r}\left(\begin{array}{l}
E_{i x} \\
E_{i y}
\end{array}\right) \\
=Q\left(\begin{array}{cc}
g_{x}^{2}\left(\delta_{y}+i \gamma_{y}+G_{x x}\right) & -g_{x} g_{y} G_{x y} \\
-g_{x} g_{y} G_{x y} & g_{y}^{2}\left(\delta_{x}+i \gamma_{x}+G_{x x}\right)
\end{array}\right)\left(\begin{array}{l}
E_{i x} \\
E_{i y}
\end{array}\right)-\left(\begin{array}{c}
E_{i x} \\
E_{i y}
\end{array}\right)
\end{gathered}
$$

where $E_{o x, y}$ indicates the output field in $x$ - and $y$-polarization, $Q=\frac{i \omega \eta_{0}}{2 a^{2}} \frac{1}{\operatorname{det} A}$. Therefore, the reflection matrix is 


$$
\begin{gathered}
\hat{r}=Q\left(\begin{array}{cc}
g_{x}^{2}\left(\delta_{y}+i \gamma_{y}+G_{x x}\right) & -g_{x} g_{y} G_{x y} \\
-g_{x} g_{y} G_{x y} & g_{y}^{2}\left(\delta_{x}+i \gamma_{x}+G_{x x}\right)
\end{array}\right)-\hat{I} \\
=\left(\begin{array}{ll}
a+i b & c+i d \\
c+i d & e+i f
\end{array}\right)
\end{gathered}
$$

where

$$
\left\{\begin{array}{c}
a=Q g_{x}^{2}\left(\delta_{y}+G_{x x}\right)-1=Q g_{x}^{2}\left(\omega-\omega_{y}+G_{x x}\right)-1 \\
b=g_{x}^{2} \gamma_{y} \\
c=-g_{x} g_{y} G_{x y} \\
d=0 \\
e=Q g_{y}^{2}\left(\delta_{x}+G_{x x}\right)-1=Q g_{y}^{2}\left(\omega-\omega_{x}+G_{x x}\right)-1 \\
f=g_{y}^{2} \gamma_{x}
\end{array}\right.
$$

According to Eq. S16, S23 and S24, we can get the real and imaginary part of the polarization converted part $r_{+-}(\boldsymbol{R})$ as

$$
\left\{\begin{array}{c}
R e\left[r_{+-}\right]=\frac{Q}{2}\left(g_{x}^{2}\left(\omega-\omega_{y}+G_{x x}\right)-g_{y}^{2}\left(\omega-\omega_{x}+G_{x x}\right)\right) \\
\operatorname{Im}\left[r_{+-}\right]=\frac{1}{2}\left(g_{x}^{2} \gamma_{y}-g_{y}^{2} \gamma_{x}+2 g_{x} g_{y} G_{x y}\right)
\end{array}\right.
$$

If there is only one resonance in either $x$ or $y$ polarization, let's say in $x$ polarization, i.e., $\omega_{x} \approx$ $\omega, \omega_{y} \approx 0$, the real part of $r_{+-}(\boldsymbol{R})$ becomes $R e\left[r_{+-}\right] \approx \frac{Q}{2}\left(g_{x}^{2}\left(\omega+G_{x x}\right)-g_{y}^{2} G_{x x}\right)=\frac{Q}{2} g_{x}^{2} \omega+$ $\frac{Q}{2} G_{x x}\left(g_{x}^{2}-g_{y}^{2}\right) \approx \frac{Q}{2} g_{x}^{2} \omega>0$. Therefore, the maximum phase of $r_{+-}(\boldsymbol{R})$ is $\pi$ as shown in fig. S8A. However, if there are two resonances in both $x$ and $y$ polarization, i.e., $\omega_{x} \approx \omega, \omega_{y} \approx \omega$, the real part of $r_{+-}(\boldsymbol{R})$ around the resonance frequency becomes $R e\left[r_{+-}\right] \approx \frac{1}{2} Q G_{x x}\left(g_{x}^{2}-g_{y}^{2}\right)$, which can be either positive or negative by rationally controlling $g_{x}$ and $g_{y}$. As a result, a full $2 \pi$ phase can be obtained as shown in fig. S8B.

Figure S9 shows the simulated results of the reflection spectrum with linear polarized incidence. When $L_{1}$ is fixed as $L_{1}=70 \mathrm{~nm}$ and the length of $L_{3}$ is controlled from $90 \mathrm{~nm}$ to 150 $\mathrm{nm}$ as shown in fig. S9A, the resonance dips in the $y$-polarization $\left(r_{y y}\right)$ keep constant as shown in the dash line because the effective length of the nanostructure in $y$-direction keeps constant. However, since the effective length of the nanostructure in $x$-direction in increasing, the resonance dips of $r_{x x}$ red shift as shown in the solid line. It can be seen that there is a cross of the two resonances in $x$ - and $y$-polarization, so that the coupling of the two polarization enables a full $2 \pi$ phase change of $r_{+-}(\boldsymbol{R})$ as discussed before. Likewise, if $L_{3}$ is fixed as $L_{3}=100 \mathrm{~nm}$ and the 
length of $L_{1}$ is controlled from $50 \mathrm{~nm}$ to $80 \mathrm{~nm}$, a resonance dip crossing is also observed as shown in fig. S9B. Therefore, by controlling $L_{1}$ and $L_{3}$, it is able to realize a full $2 \pi$ phase change of $r_{+-}(\boldsymbol{R})$.

The simulated results of real and imaginary part of $r_{+-}(\boldsymbol{R})$ in the parameter space covered by $L_{1} \in[48 \mathrm{~nm}, 58 \mathrm{~nm}]$ and $L_{3} \in[112 \mathrm{~nm}, 122 \mathrm{~nm}]$ are shown in fig. S10. The yellow and blue areas represent the positive and negative sign of $r_{+-}$. An exceptional point is obtained as indicated in the red star, where the eigenstates degenerate as RCP $(|-\rangle)$, such that $r_{+-}=0$. At the vicinity of the exceptional point, when $L_{1}$ increases, the resonance frequency $\omega_{y}$ decreases while $\omega_{x}$ keeps constant around $\omega$ as shown in fig. S9B, so that the real part of $r_{+-}: \operatorname{Re}\left[r_{+-}\right]=\frac{1}{2}\left(Q g_{x}^{2}\left(\omega-\omega_{y}+\right.\right.$ $\left.\left.10 G_{x x}\right)-Q g_{y}^{2}\left(\omega-\omega_{x}+G_{x x}\right)\right) \approx \frac{1}{2} Q g_{x}^{2}\left(\omega-\omega_{y}\right)$ increases, resulting in a positive value of $\operatorname{Re}\left[r_{+-}\right]$with larger $L_{1}$ as shown in fig. S10A, and vice-versa. When $L_{3}$ increases, $g_{x}, G_{x y}$ and $\gamma_{x}$ are increasing, the imaginary part of $r_{+-}: \operatorname{Im}\left[r_{+-}\right]=\frac{1}{2}\left(g_{x}^{2} \gamma_{y}-g_{y}^{2} \gamma_{x}+2 g_{x} g_{y} G_{x y}\right)$ increases. Because the power index of the first term $\left(g_{x}^{2}\right)$ and third term $\left(g_{x} G_{x y}\right)$ is twice compared to the second term $\left(\gamma_{x}\right)$, such that the increasing of $g_{x}^{2} \gamma_{y}+2 g_{x} g_{y} G_{x y}$ is more dramatic than the decreasing of $g_{y}^{2} \gamma_{x}$. Thus, a positive value of $\operatorname{Im}\left[r_{+-}\right]$is obtained with larger $L_{3}$ as shown in fig. $\mathrm{S} 10 \mathrm{~B}$, and vice-versa. Therefore, a full $2 \pi$ phase of $r_{+-}$is obtained by encircling the EP in the parameter space of $\left(L_{1}, L_{3}\right)$ at the vicinity of EP. As discussed in Note 2 , such $2 \pi$ phase is topologically protected with arbitrary closed path that is encircling the EP, even if we extend the parameter space of $\left(L_{1}, L_{3}\right)$ to $L_{1} \in(0 \mathrm{~nm}, 100 \mathrm{~nm}), L_{3} \in(50 \mathrm{~nm}, 250 \mathrm{~nm})$ as shown in Fig. 1B and $3 \mathrm{C}$ in the main text and fig. S11, as long as there is only one EP in the interested parameter space. 

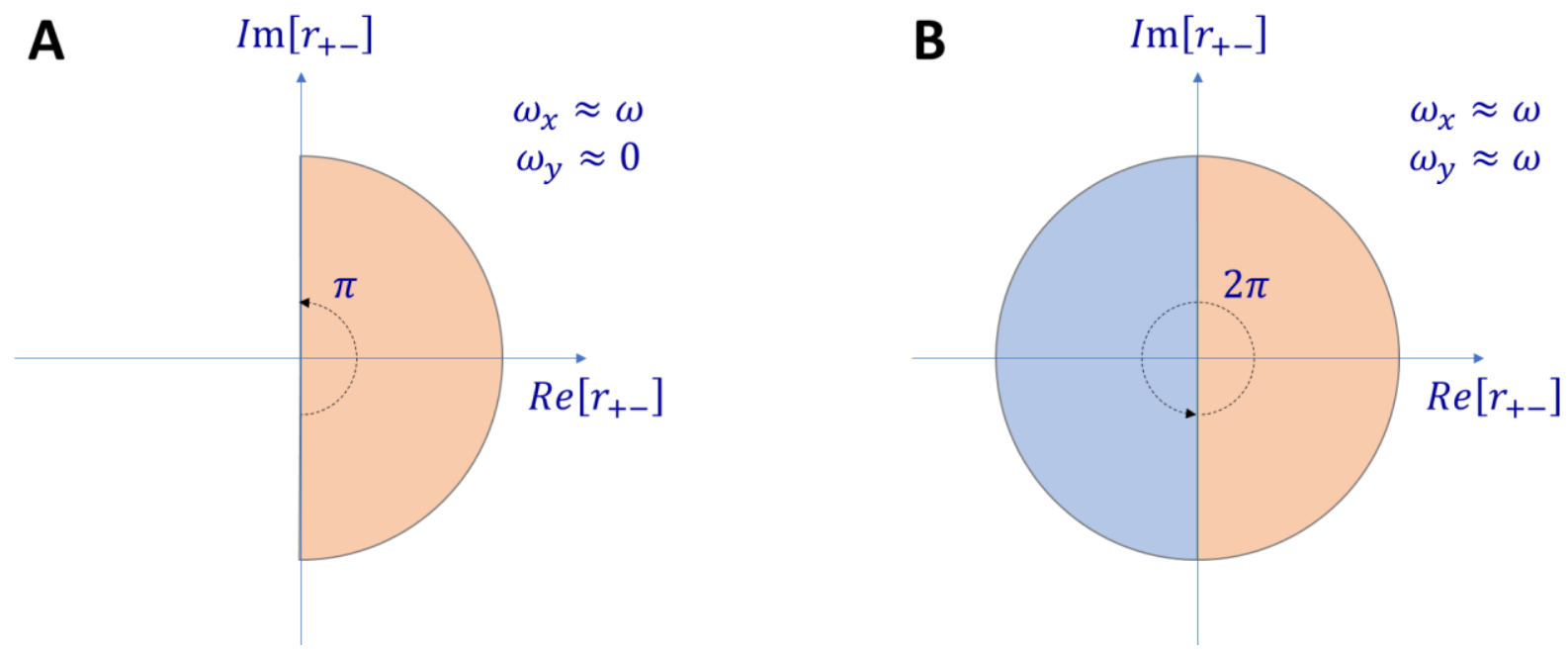

Fig. S8. Phase accumulation of $\boldsymbol{r}_{+-}$from coupled-mode theory. (A) When there is only one resonance in $x$-polarization $\left(\omega_{x} \approx \omega, \omega_{y} \approx 0\right)$, the maximum phase accumulation of $r_{+-}$is $\pi$. (B) Together with the other resonance in $y$-polarization $\left(\omega_{x} \approx \omega, \omega_{y} \approx \omega\right)$, a full $2 \pi$ phase can be achieved. 

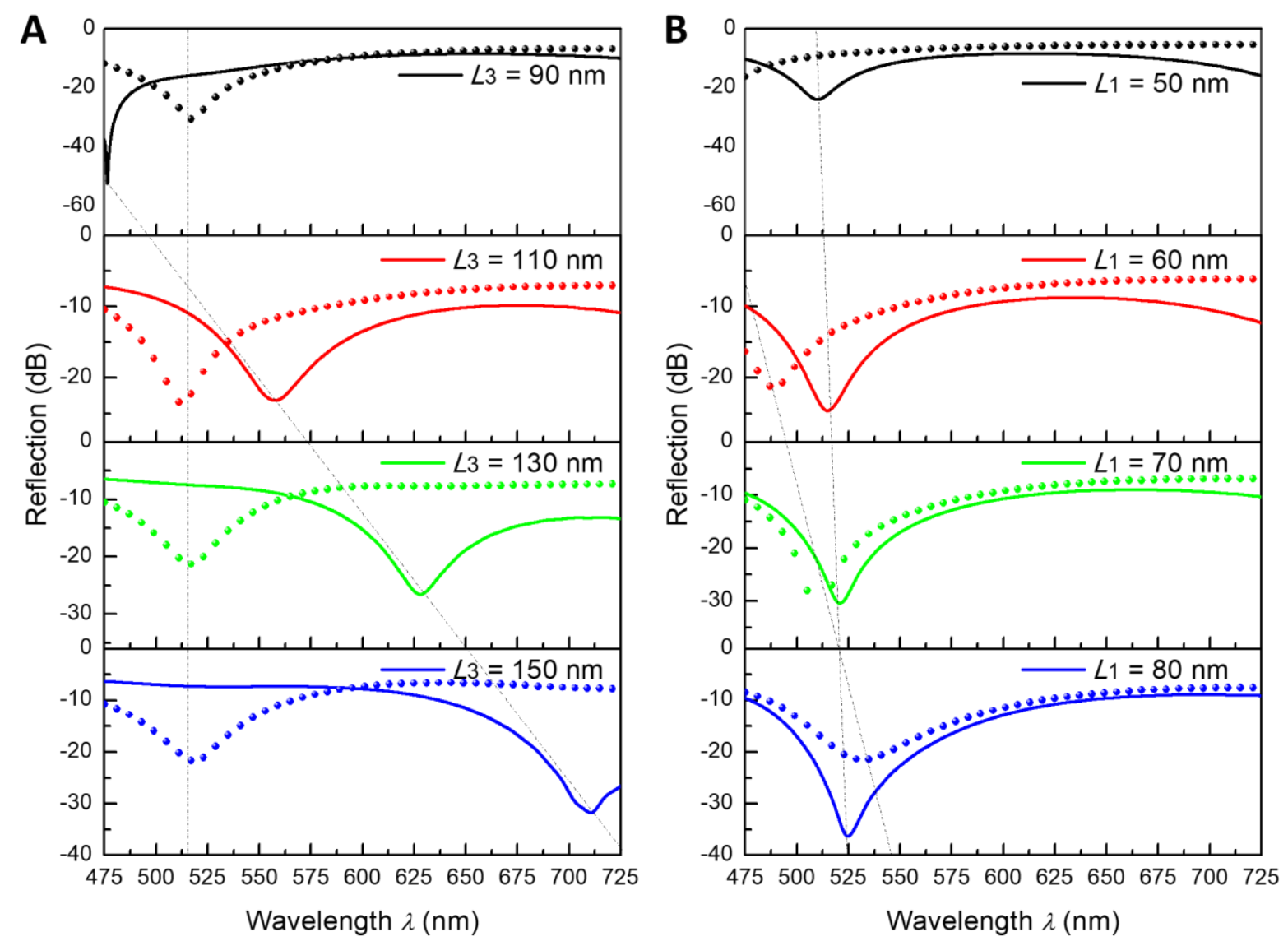

Fig. S9. Simulated results of the reflection spectrum with linear polarization incidence. Solid and dot lines represent $r_{x x}$ and $r_{y y}$, respectively. (A) Simulated results with $L_{1}$ fixed as $70 \mathrm{~nm}$ and $L_{3}$ is changed from $90 \mathrm{~nm}$ to $150 \mathrm{~nm}$. The effective length of the meta-atom in the $y$-direction is fixed, such that the resonance dips of $r_{y y}$ keep constant. While the effective length of the metaatom in the $x$-direction is increasing, resulting in a red shift of the resonance dips of $r_{x x}$. (B) Simulated results with $L_{3}$ fixed as $100 \mathrm{~nm}$ and $L_{1}$ is changed from $50 \mathrm{~nm}$ to $80 \mathrm{~nm}$. The effective length of the meta-atom in the $x$-direction is fixed, such that the resonance dips of $r_{x x}$ keep constant. While the effective length of the meta-atom in the $y$-direction is increasing, resulting in a red shift of the resonance dips of $r_{y y}$. It can be observed that the two resonance dips in $x$ - and $y$ directions have a cross as shown in the two dash lines. Therefore, by changing $L_{1}$ and $L_{3}$, it is possible to couple the two resonances in $x$ - and $y$ - directions to obtain a full $2 \pi$ phase of $r_{+-}$. 

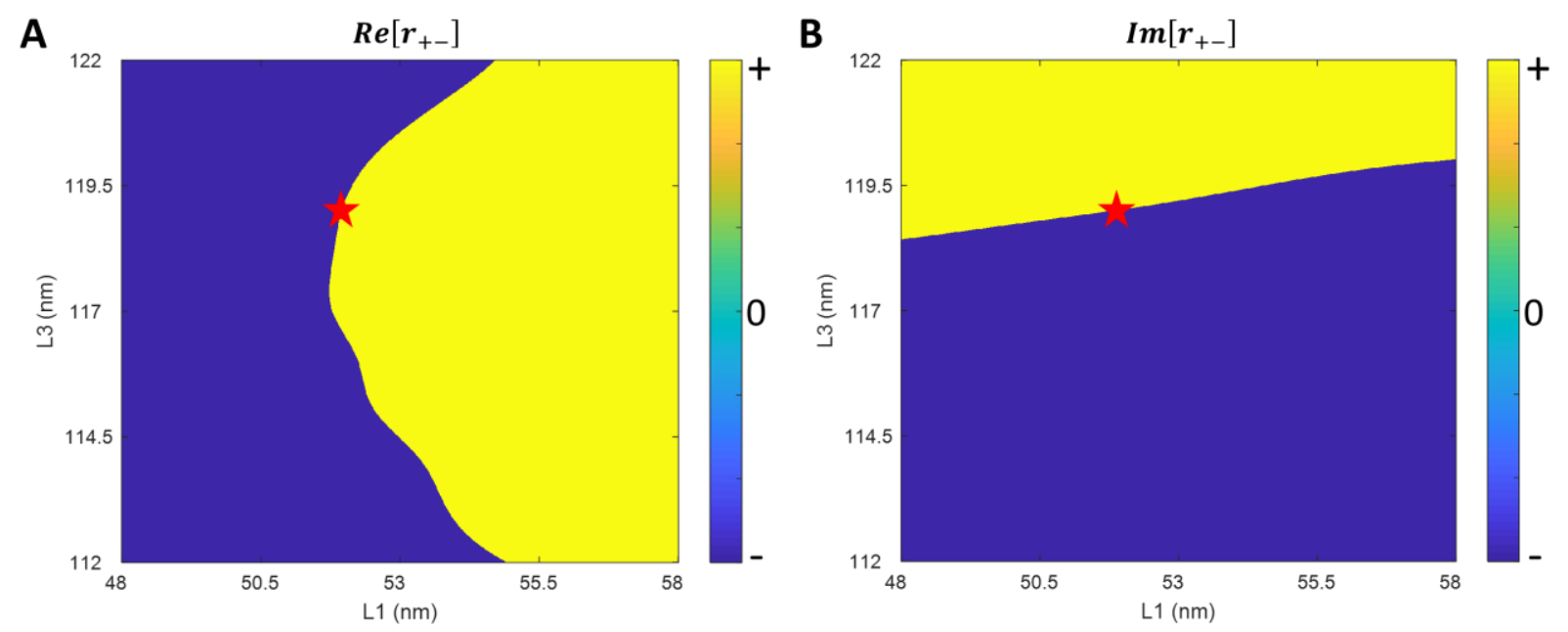

Fig. S10. Simulated results of $r_{+-}$in the parameter space covered by $L_{1} \in[48 \mathrm{~nm}, 58 \mathrm{~nm}]$ and $L_{3} \in[112 \mathrm{~nm}, 122 \mathrm{~nm}]$. The yellow and blue colors represent the positive and negative sign of $r_{+-}$, respectively. The red dots represent the exceptional point, where both real and imaginary parts of $r_{+-}$vanish. (A) Real and (B) imaginary part of $r_{+-}$. At the vicinity of the EP $\left(\operatorname{Re}\left[r_{+-}\right]=\right.$ $\left.\operatorname{Im}\left[r_{+-}\right]=0\right)$, for the real part of $r_{+-}$, when $L_{1}$ increases, the resonance frequency $\omega_{y}$ decreases, so that $\operatorname{Re}\left[r_{+-}\right]$increases, resulting in a positive value of $\operatorname{Re}\left[r_{+-}\right]$, and vice-versa. For the imaginary part of $r_{+-}$, when $L_{3}$ increases, both $g_{x}$ and $G_{x y}$ increases, so that $\operatorname{Im}\left[r_{+-}\right]$increases, resulting in a positive value of $\operatorname{Im}\left[r_{+-}\right]$, and vice-versa. Therefore, a full $2 \pi$ phase of $r_{+-}$is obtained by encircling the EP. 

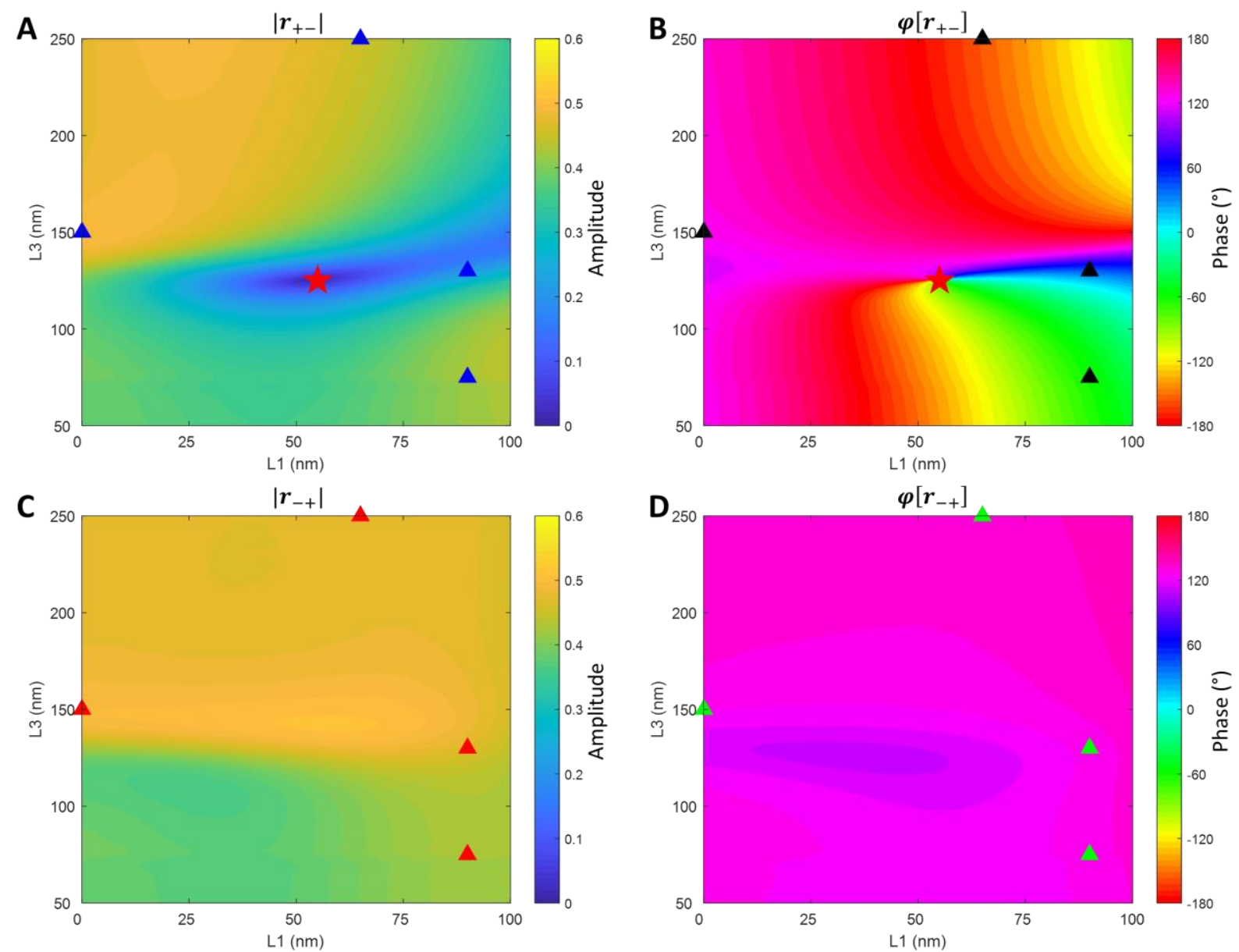

Fig. S11. Simulated reflection map of topological metasurface at the wavelength of $600 \mathrm{~nm}$.

(A) Amplitude and (B) phase of $r_{+-}$. (C) Amplitude and (D) phase of $r_{-+}$in the parameter space covered by $L_{1} \in[0,100 \mathrm{~nm}]$ and $L_{3} \in[50 \mathrm{~nm}, 250 \mathrm{~nm}]$. The EP occurs at the parameter of $\left(L_{1}, L_{3}\right)=(52 \mathrm{~nm}, 119 \mathrm{~nm})$ as highlighted in red stars, where the amplitude of $r_{+-}$vanishes and a vortex phase profile of $\varphi\left[r_{+-}\right]$is observed. A full $2 \pi$ phase accumulation can be obtained by encircling the EP. The corresponding 3D surf maps of $r_{ \pm \mp}$ are shown in Fig. 1B-1C in the main text. Figure S11B and S11D are the same with Fig. 3C and 3D in the main text. The four triangles represent four metasurface design with an ET phase interval of $90^{\circ}$. The amplitude and phase values of these four designs are shown in Fig. 3G in the main text. 

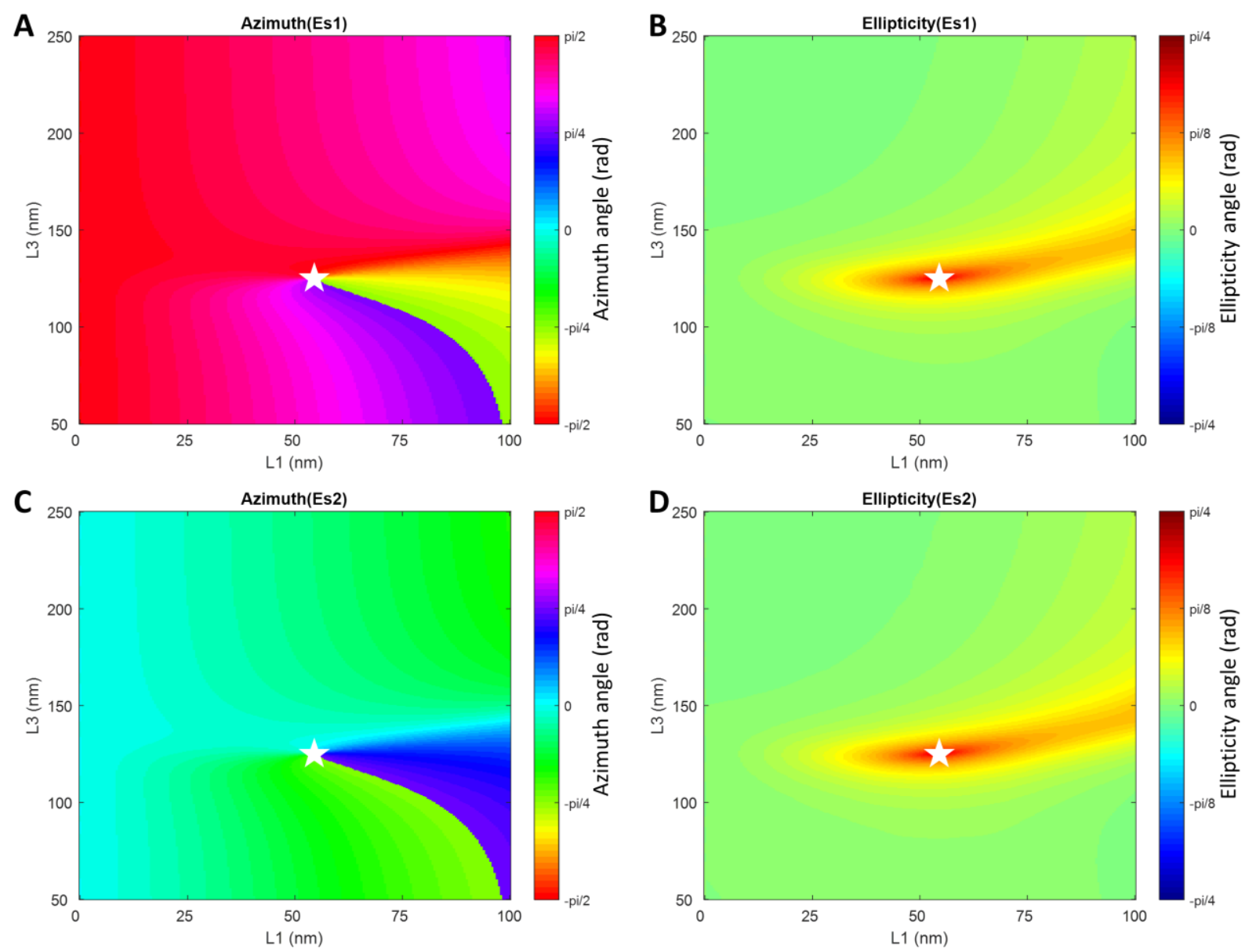

Fig. S12. Eigenstates of reflection matrix at the wavelength of $600 \mathrm{~nm}$. (A) Azimuth and (B) ellipticity angle of the first eigenstate. (C) Azimuth and (D) ellipticity angle of the second eigenstate in the parameter space covered by $L_{1} \in[0,100 \mathrm{~nm}]$ and $L_{3} \in[50 \mathrm{~nm}, 250 \mathrm{~nm}]$. The red stars represent the EPs where both eigenstates coalesce as RCP. 
A

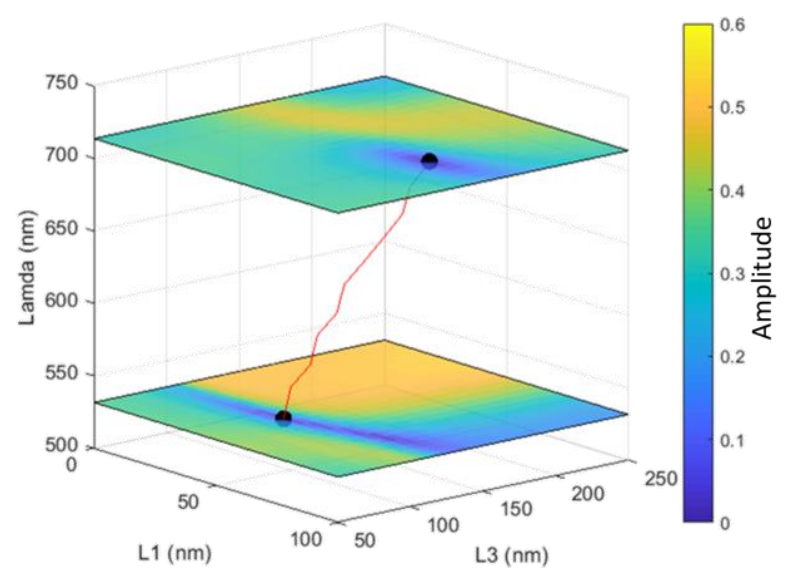

B $\varphi\left[r_{+-}\right]$

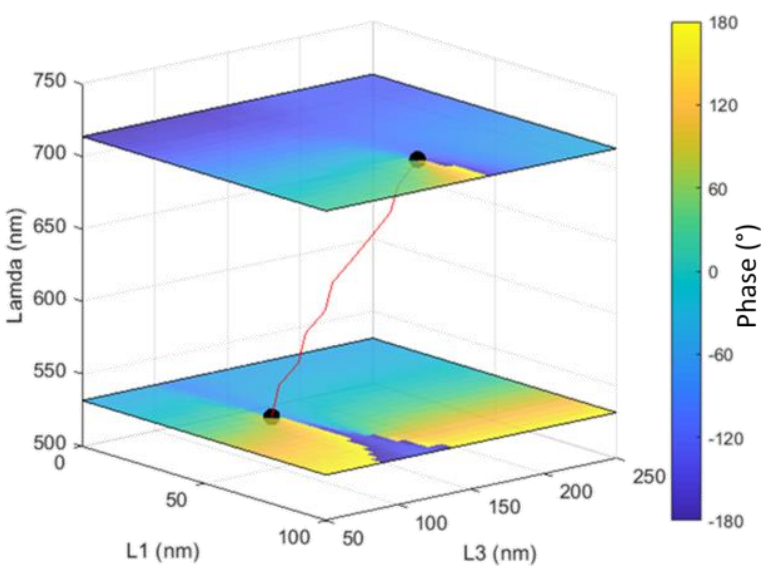

Fig. S13. Exceptional point trajectory as a function of wavelength in the parameter space of $\left(\boldsymbol{L}_{1}, \boldsymbol{L}_{2}\right)$. (A) Amplitude and (B) phase of $r_{+-}$. The red lines represent the trajectory of the EP. 


\section{Supplementary Note 5: Combination with Pancharatnam-Berry (PB) phase by rotating the metasurface.}

Assume the metasurface is rotated with an angle of $\theta$ relative to the reference coordinate system $\{x, y\}$ (see Fig. 1D in the main text), the reflection matrix in the circular-polarization representation can be expressed as,

$$
\hat{r}^{\theta}(\boldsymbol{R})=\left(\begin{array}{cc}
r_{++}^{\theta}(\boldsymbol{R}) & r_{+-}^{\theta}(\boldsymbol{R}) \\
r_{-+}^{\theta}(\boldsymbol{R}) & r_{--}^{\theta}(\boldsymbol{R})
\end{array}\right)=M(-\theta) \hat{r}(\boldsymbol{R}) M(\theta)
$$

By employing the Pauli matrices notation $\left\{\sigma_{1}, \sigma_{2}, \sigma_{3}\right\}$ and the identity matrix $\hat{I}(29)$, the $2 \times$ 2 rotation matrix $M$ is equal to $e^{i \theta \sigma_{3}}$, which can be expanded by Taylor series as $M(\theta)=\cos \theta \hat{I}+$ $i \sin \theta \sigma_{3}$. Therefore, the reflection matrix can be described as following,

$$
\begin{gathered}
\hat{r}^{\theta}(\boldsymbol{R})=\frac{1}{2}\left(r_{x x}+r_{y y}\right) \hat{I}+\frac{i}{2}\left(r_{x y}-r_{y x}\right) \sigma_{3} \\
+\frac{1}{2}\left(r_{x x}-r_{y y}\right)\left(e^{-i 2 \theta} \sigma_{+}+e^{i 2 \theta} \sigma_{-}\right)+\frac{i}{2}\left(r_{x y}+r_{y x}\right)\left(-e^{-i 2 \theta} \sigma_{+}+e^{i 2 \theta} \sigma_{-}\right)
\end{gathered}
$$

where two spin-flip operators $\sigma_{ \pm}=\frac{\sigma_{1} \pm i \sigma_{2}}{2}$ satisfy $\sigma_{ \pm}| \pm\rangle=0$ and $\sigma_{ \pm}|\mp\rangle=| \pm\rangle$, with $| \pm\rangle$ denoting LCP and RCP, respectively. When the metasurface is illuminated with RCP $(|-\rangle)$, the reflection is

$$
\begin{aligned}
r^{\theta}(\boldsymbol{R})=\hat{r}^{\theta}(\boldsymbol{R})|-\rangle= & \frac{1}{2}\left(r_{x x}+r_{y y}\right) \hat{I}|-\rangle+\frac{i}{2}\left(r_{x y}-r_{y x}\right) \sigma_{3}|-\rangle \\
& +\frac{1}{2}\left(r_{x x}-r_{y y}\right) e^{-i 2 \theta}|+\rangle-\frac{i}{2}\left(r_{x y}+r_{y x}\right) e^{-i 2 \theta}|+\rangle
\end{aligned}
$$

where the first two terms represent the CP unconverted part and the last two terms represent the $\mathrm{CP}$ converted part, i.e.,

$$
r_{+-}^{\theta}(\boldsymbol{R})=\frac{\left(r_{x x}-r_{y y}\right)-i\left(r_{x y}+r_{y x}\right)}{2} e^{-i 2 \theta}=r_{+-}^{0}(\boldsymbol{R}) e^{-i 2 \theta}
$$

where $r_{+-}^{0}(\boldsymbol{R})$ represents the original ET phase without rotation as shown in Eq. S15. Therefore, a geometric phase of $-2 \theta$ is applied into $r_{+-}$when the metasurface is rotated with an angle of $\theta$. Similarly, when the metasurface is illuminated with LCP $(|+\rangle)$, the CP converted part can be expressed as,

$$
r_{-+}^{\theta}(\boldsymbol{R})=\frac{\left(r_{x x}-r_{y y}\right)+i\left(r_{x y}+r_{y x}\right)}{2} e^{i 2 \theta}=r_{-+}^{0}(\boldsymbol{R}) e^{i 2 \theta}
$$

Thus, an opposite geometric phase of $2 \theta$ is applied into $r_{-+}$.

In conclusion, the reflective $\mathrm{CP}$ conversion is, 


$$
r_{ \pm \mp}^{\theta}(\boldsymbol{R})=r_{ \pm \mp}^{0}(\boldsymbol{R}) e^{\mp i 2 \theta}=\left|r_{ \pm \mp}^{0}(\boldsymbol{R})\right| e^{i \varphi\left[r_{ \pm \mp}^{0}(\boldsymbol{R})\right]} e^{\mp i 2 \theta}
$$

where $|x|$ and $\varphi[x]$ represent the amplitude and phase of complex value $x$. Therefore, the overall phase of the $\mathrm{CP}$ conversion term with rotation angle of $\theta$ is the combination of the original ET phase without rotation and a geometric phase as,

$$
\varphi\left[r_{ \pm \mp}^{\theta}(\boldsymbol{R})\right]=\varphi\left[r_{ \pm \mp}^{0}(\boldsymbol{R})\right] \mp 2 \theta
$$

The simulated reflection 3D surf maps of the rotated metasurface with $\left(L_{1}, L_{2}\right)$ fixed at (80 nm, 95 $\mathrm{nm}$ ) when the rotation angle of the meta-atom is changed from 0 to $2 \pi$ are shown in Fig. $1 \mathrm{E}$ and $1 \mathrm{~F}$ in the main text. The corresponding 2D color maps are shown in fig. S14. The simulated PB phase is also presented in Fig. $3 \mathrm{~F}$ and $3 \mathrm{G}$ in the main text. An opposite of PB phase of $\mp 2 \theta$ is shown in $r_{ \pm \mp}$.

In the real metasurface design, assuming the desired phase profile for $r_{ \pm \mp}^{\theta}$ is $\varphi_{d}\left[r_{ \pm \mp}^{\theta}\right]$, we can derive the necessary ET phase of $r_{+-}^{0}$ and the necessary rotation angles corresponding to the additional PB phase, as given by Eq. S32 (where we have taken into account that $\varphi\left[r_{-+}^{0}\right]=0$ ),

$$
\left\{\begin{array}{c}
\varphi\left[r_{+-}^{0}\right]=\varphi_{d}\left[r_{+-}^{\theta}\right]+\varphi_{d}\left[r_{-+}^{\theta}\right] \\
\theta=\frac{\varphi_{d}\left[r_{-+}^{\theta}\right]}{2}
\end{array}\right.
$$



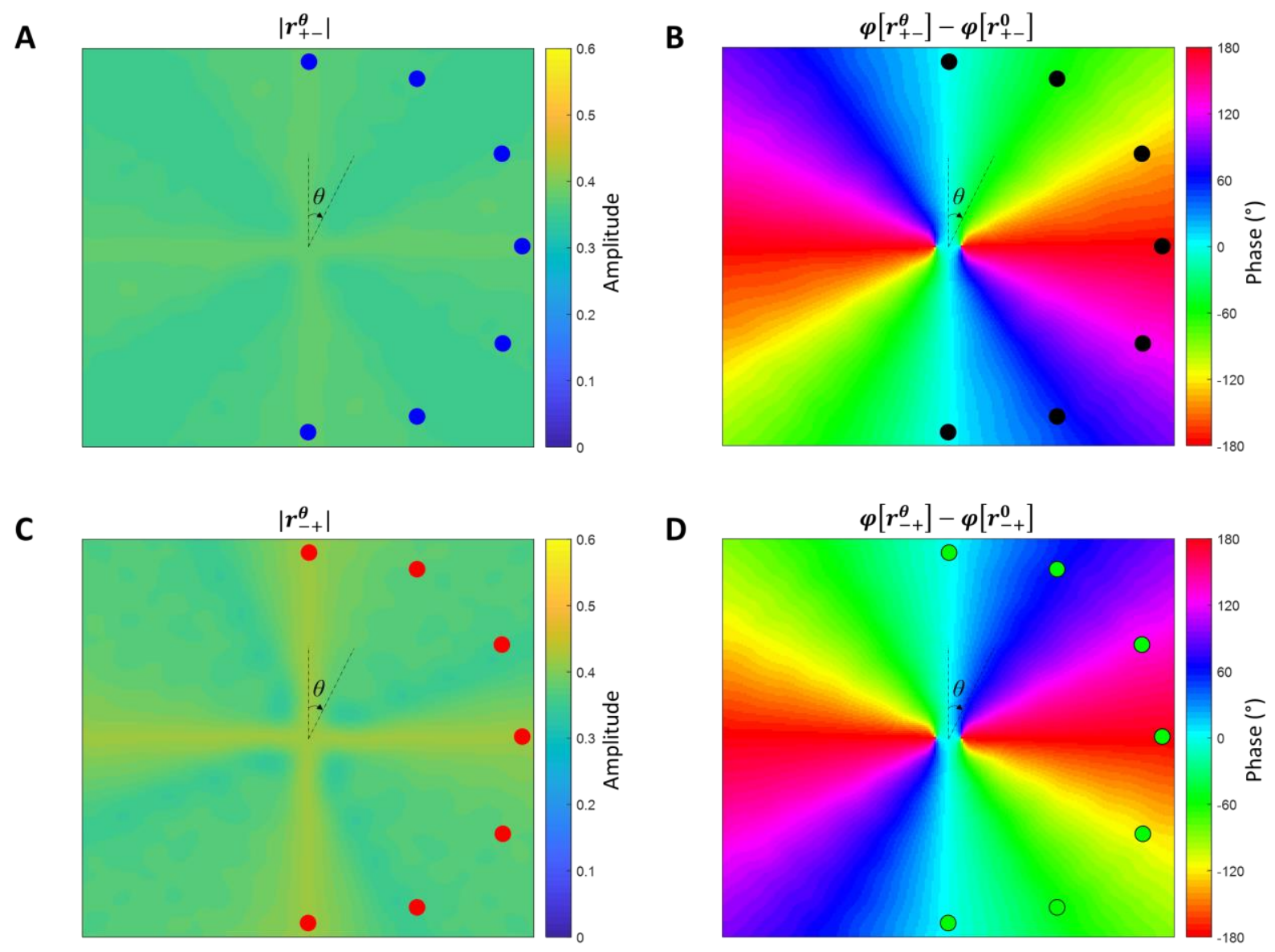

Fig. S14. Simulated reflection color maps of rotated metasurface at the wavelength of 600 $\mathrm{nm}$ with $\left(L_{1}, L_{2}\right)$ fixed at $(80 \mathrm{~nm}, 95 \mathrm{~nm})$ when the rotation angle of the meta-atom is changed

from 0 to $2 \pi$. (A) Amplitude and (B) phase of $r_{+-}$. (C) Amplitude and (D) phase of $r_{-+}$with rotation angle of $\theta$ ranging from 0 to $2 \pi$. The represented phase of $r_{ \pm \mp}^{\theta}$ in $\mathrm{B}$ and $\mathrm{D}$ has been subtracted by the phase of $r_{ \pm \mp}^{0}$ with zero rotation angle, such that a pure PB phase can be observed. An opposite topology of vortex phase profile can be seen, which is intrinsically different from the topological phase by encircling the EP as shown in fig. S11. The corresponding 3D surf maps of $r_{ \pm \mp}^{\theta}$ are shown in Fig. 1E-1F in the main text. Figure S14B and S14D are the same with Fig. 3E and $3 \mathrm{~F}$ in the main text. The seven dots represent seven rotation angles from $0^{\circ}$ to $180^{\circ}$ with a step of $30^{\circ}$. The amplitude and phase values of these seven designs are shown in Fig. $3 \mathrm{H}$ in the main text. 